\title{
Decoherence in quantum cavities: Environmental erasure of carpet-type structures
}

\author{
E. Honrubid ${ }^{*}$ and A. S. Sand \\ Department of Optics, Faculty of Physical Sciences, Universidad Complutense de Madrid \\ Pza. Ciencias 1, Ciudad Universitaria - 28040 Madrid, Spain
}

(Dated: June 14, 2021)

\begin{abstract}
The interaction with an environment provokes decoherence in quantum systems, which gradually suppresses their capability to display interference traits. Hence carpet-type structures, which arise after the release of a localized state inside a quantum cavity, constitute an ideal laboratory to study and analyze the robustness of the interference process that underlies this phenomenon against the harmful effects of decoherence. Such a released localized state may represent a radiation mode inserted into a multimode interference device or a cold-atom system released in an optical trap, for instance. Here, without losing any generality, for simplicity, the case of a particle with a mass $m$ is considered and described by a localized state corresponding to the ground state of a square box of width $w$, which is released inside a wider cavity (with a width $L>w$ ). The effects of decoherence are then numerically investigated by means of a simple dynamical model that captures the essential features of the phenomenon under Markovian conditions, leaving aside extra complications associated with a more detailed dynamical description of the system-environment interaction. As it is shown, this model takes into account and reproduces the fact that decoherence effects are stronger as energy levels become more separated (in energy), which translates into a progressive collapse of the energy density matrix to its main diagonal. However, because energy dissipation is not considered, an analogous behavior is not observed in the position representation, where a proper spatial localization of the probability density does not take place, but rather a delocalized distribution. This result emphasizes the fact that classicality is reached only if both decoherence and dissipation coexist; otherwise, non-classical traits might still persist. Actually, as it is also shown, in the position representation some off-diagonal correlations indeed survive unless an additional spatial-type factor is included in the model. This makes evident the rather complex nature of the decoherence phenomenon and hence the importance to have a familiarity with how it manifests in different representations, particularly with the purpose to determine and design reliable control mechanisms.
\end{abstract}

\section{INTRODUCTION}

Decoherence is central to the description and understanding of quantum systems whenever they are not under ideal isolation conditions [1. Their interaction with other surrounding systems leads them to gradually lose their coherence properties and hence to exhibit behaviors that resemble those typical of classical systems. This effect has been commonly referred to in the literature as the emergence of the classical world 28 . Decoherence is ubiquitous in a myriad of systems and applications [9], e.g., quantum dots [10 12, quantum game theory [13. 14, quantum walks [15, 16, quantum information [17 20], two-level systems [21 23], cavities [24 26], ion trapping [27] or the spin-boson model [9, 28]. Similarly different models have been proposed to study and quantify its effects on the coherence of quantum systems as well as to control them 29 39]. Furthermore, a number of experiments have also been conduced in recent year to test such models at a fundamental level [40,43].

Depending on the system or, to be more precise, the nature and strength of its interaction with a surrounding environment (many times also on the intrinsic properties of this environment), different alternative theoreti-

\footnotetext{
* efrenhon@ucm.es

$\dagger \overline{\text { a.s.sanz@fis.ucm.es }}$
}

cal models can be used to model the effects of decoherence [1, 9]. These models may consist of simple sets of differential equations in terms of energy levels, such as the Bloch equations, or more general equations of motion, such as the Lindblad equation, valid in any representation. However, taking such theoretical models and their outcomes as the basis, it is still possible to build simpler phenomenological models that capture the physical essence of the phenomenon and, by adjusting a few parameters, also provide us with a clear picture of the processes involved at different levels of detail. This is of particular interest in the analysis of highly intricate structures generated by interference, as it is the case of quantum carpets, which generate inside cavities by linearly and coherently superimposing a number of energy eigenstates [44 47]. It is clear that, as the number of eigenstates increases and the frequencies involved in the superposition become higher and higher, the system becomes more sensitive to decoherence. Hence quantum carpets seem to constitute an ideal scenario to explore the effects of decoherence [48, 49].

In this work we analyze the consequences of a purely decoherent model on the erasure of quantum carpets in terms of the symmetries displayed by the latter. More specifically, here we consider the carpets developed upon the release of an initially localized matter waved describing a particle with mass $m$ for simplicity, although without any loss of generality, since the treatment can equally 
be applied to light carpets inside confining cavities (resonant cavities or multimode interference devices). This analysis, performed in the position representation as well as in the energy representation, renders an interesting picture on how decoherence acts in each case. Eventually this serves to better understand more refined and exact models, where such splitting is not possible due to their intrinsic formal and conceptual nature. Under Markovian conditions decoherence has important effects on both the position and the energy representations [50]. Thus, the model considered here relies on these observations and consists of a simple Markovian coherence-damping term where energy differences between eigenstates and twopoint position correlations appear separately. As it is shown, this leads to a bare addition of level populations. In the energy representation, this translates into a gradual suppression of the off-diagonal elements of the density matrix, only surviving the elements of the main diagonal, i.e., the elements that physically account for the populations. In the position representation, on the other hand, it is observed that the probability density approaches a nonhomogeneous, delocalized density distribution along the cavity. Furthermore, in terms of the density matrix in the position representation, it is also noticed that the probability accumulates not only along the main diagonal, but also along the secondary diagonal when the initial state displays an even symmetry with respect to the center of the cavity. In order to remove such a nonphysical behavior, associated with the symmetry of the system, an additional decoherence term depending on twopoint correlations has to be explicitly taken into account, which explains the typical exponential decays in terms of factors of the form $\left(x-x^{\prime}\right)^{2}$ that appear in spatial decoherence models 6]. Furthermore, in order to provide a clearer picture of the decoherence dynamics, i.e., the transition from a highly organized interference-mediated structure to a stationary (equilibrium) state due to decoherence, the generation of the quantum carpet has also been monitored with the aid of Bohmian trajectories, which have already been used to explore analogous effects in the context of the two-slit experiment [51,53.

The work is organized as follows. Section [II deals with the theoretical treatment of the time evolution of localized states or signals freely released in the cavity and the subsequent emergence of quantum carpets. It also includes a brief overview of the Bohmian-type methodology that will be used to visualize and hence to better understand the evolution of the probability distribution in terms of density streamlines or Bohmian trajectories [54, 55]. Furthermore, several cases of symmetric and asymmetric carpets are presented and discussed with the purpose to serve later on to evaluate the effects of decoherence. In particular, the carpets considered arise from the time-evolution of single (symmetrically and asymmetrically) localized signals and coherent superpositions made of two initially (and symmetrically) localized signals. In Sec. IIIthe decoherence model is introduced from the standard point of view and also within the Bohmian context; the effects of this model on quantum carpets are then analyzed and discussed in both the position representation and the energy representations. We conclude with a summary and discussion in Sec. IV.

\section{QUANTUM CARPET DYNAMICS}

\section{A. General aspects}

Quantum carpets, the highly symmetric pattern displayed in both space and time by the probability density inside a cavity, arise as a consequence of a rather complex interference process involving a number of energy eigenstates (vibrational modes) of the cavity [47]. This is the behavior, for instance, that follows after pumping a localized state into a cavity and then letting it freely evolve inside such a cavity. The state can be a mode propagated along an optical fiber and then released into a broader cavity, such as a multimode interference device [56 58 , or a cold-atom system confined and guided in an optical trap [59]. In either case, as soon as the localized wave describing the state of the system is released inside the wider cavity, it starts reconfiguring according to the new boundaries, thus giving rise to the appearance of interference traits, which depend on the shape of the initially localized state and the size of the cavity [60. To better understand the process and also to be self-contained, let us start by briefly describing the process that leads to the appearance of quantum carpets as well as some of its most relevant properties in connection to this work.

Thus, consider that the matter wave associated with a particle with mass $m$ is pumped into a cavity of a certain width, which will be assumed to be one dimensional for simplicity. Such a matter wave is describable in terms of a localized wave function, which will be referred to from now on as the input signal, making use of a more operational description, also valid in the optical scenario 61. Note that this input signal describes the particular shape of the input beam, which can be a single vibrational mode or eigenstate transported from a narrower cavity (waveguide) to the new one. This is the case here considered, where the, say, collecting cavity is assumed to be a square box centered at $x=0$ and with a width $L$, larger than the typical width associated with the input signal, henceforth denoted by $w$.

As it is well known, in such a case, the input signal can then easily be recast as a coherent superposition of the corresponding basis set of energy modes (eigenstates) $\left\{\varphi_{\alpha}(x) \in \mathbb{R}, \alpha=0,1,2, \ldots,|x| \leq L / 2\right\}$ as

$$
\psi_{0}(x)=\sum_{\alpha} c_{\alpha} \varphi_{\alpha}(x)
$$

Each coefficient $c_{\alpha}$ is obtained from the projection of the input signal onto the corresponding mode, i.e.,

$$
c_{\alpha}=\int \varphi_{\alpha}^{*}(x) \psi_{0}(x) d x .
$$


If these coefficients are recast in polar form, i.e., as $c_{\alpha}=\left|c_{\alpha}\right| e^{i \delta_{\alpha}}$, it is clear that they contain information about how much each mode contributes to the superposition, with the weight of such a contribution given by $\left|c_{\alpha}\right|^{2}$. Hence, from now on, because they indicate the population of each mode, we will refer to them as the populations, also in compliance with the convention commonly used in level systems. In the same way, the crossed terms, $c_{\alpha} c_{\alpha^{\prime}}^{*}$, will be referred to as coherences, since they carry information about the mutual correlation between different pairs of modes. Since each coefficient carries a time-independent relative phase $\delta_{\alpha}$, they will contribute to introduce relative phase differences among different modes, thus generating constructive or destructive interference among them. As for the modes, they are simple sinusoidal functions with even and odd parity 62

$$
\begin{aligned}
& \varphi_{\alpha}^{e}(x)=\sqrt{\frac{2}{L}} \cos \left(k_{\alpha} x\right), \\
& \varphi_{\alpha}^{o}(x)=\sqrt{\frac{2}{L}} \sin \left(k_{\alpha} x\right),
\end{aligned}
$$

with $k_{\alpha}=\alpha \pi / L$, where $\alpha=2 n-1$ for the even-parity solutions $(e)$ and $\alpha=2 n$ for the odd-parity solutions (o), with $n=1,2, \ldots$ in both cases. The corresponding eigenenergies are $E_{\alpha}=\hbar^{2} k_{\alpha}^{2} / 2 m=\hbar^{2} \pi^{2} \alpha^{2} / 2 m L^{2}$.

The spectral decomposition accounted for by Eq. (1) enables a simple description of the evolution of the input signal at any subsequent time. As it is well known, because each mode is associated with a specific energy $E_{\alpha}$, the time evolution of Eq. (1) is analytical and has the simple functional form

$$
\psi(x, t)=\sum_{\alpha} c_{\alpha} \varphi_{\alpha}(x) e^{-i E_{\alpha} t / \hbar} .
$$

Due to the extra complex factors $\exp \left(-i E_{\alpha} t / \hbar\right)$, as soon as $t$ changes, all spectral components start vibrating, thus changing their local value, which translates in the aforementioned complex interference process, with probability distributions varying from time to time. However, although at some times the probability density might not keep any resemblance with the original one, at other particular times it is possible to observe the appearance of recurrences (a number of identical copies of the initial probability density distributed across the cavity) and revivals (a full reconstruction of the initial probability density).

The emergence of recurrences and revivals is better appreciated by explicitly computing the probability density associated with (4),

$$
\begin{aligned}
\rho(x, t) & =\sum_{\alpha}\left|c_{\alpha}\right|^{2} \varphi_{\alpha}^{2}(x) \\
& +\sum_{\alpha^{\prime}>\alpha}\left|c_{\alpha}\right|\left|c_{\alpha^{\prime}}\right| \varphi_{\alpha}(x) \varphi_{\alpha^{\prime}}(x) \cos \left(\omega_{\alpha \alpha^{\prime}} t-\delta_{\alpha \alpha^{\prime}}\right),
\end{aligned}
$$

where

$$
\omega_{\alpha \alpha^{\prime}} \equiv \frac{E_{\alpha^{\prime}}-E_{\alpha}}{\hbar}=2 \pi\left(\frac{\pi \hbar}{4 m L^{2}}\right)\left(\alpha^{\prime 2}-\alpha^{2}\right)
$$

and $\delta_{\alpha \alpha^{\prime}} \equiv \delta_{\alpha^{\prime}}-\delta_{\alpha}$, with $\alpha^{\prime}>\alpha$. Note that the splitting in (5) makes explicit the contribution arising from populations and coherences independently. As it can be seen, this splitting is rather convenient, because all the dynamics is contained in the coherences; populations remain unchanged unless dissipation is present, which is not the case here. Our decoherence model will focus on the second term of Eq. (5), which defines the phase relations between the different modes through the relative phase shifts $\delta_{\alpha \alpha^{\prime}}$. In the latter regard, since the input signal has no transverse displacement component, we have $\delta_{\alpha \alpha^{\prime}}=0$ for all $\alpha$ and $\alpha^{\prime}$. Those displacements imply the presence of extra factors, of the kind $e^{ \pm i k x}$ in $\psi_{0}(x)$, which eventually turn into nonvanishing relative phase shifts. However, this will not be the case here, where input signals are assumed to be pumped into the cavity without lateral motion.

In spite of the complex interference process described by (5) and the shape displayed by the input signal, it is easy to see that there is a revival of the state after some time. More specifically, this happens whenever time is such that the time-dependent phase factor $\omega_{\alpha \alpha^{\prime}} t$ is an integer multiple of $2 \pi$ for all $\alpha$ and $\alpha^{\prime}$. If we denote by $T_{\text {rev }}$ the first time $t$ at which this condition is satisfied, then we find that

$$
\omega_{\alpha \alpha^{\prime}} T_{\mathrm{rev}}=2 \pi\left(\frac{\pi \hbar}{4 m L^{2}}\right)\left(\alpha^{\prime 2}-\alpha^{2}\right) T_{\mathrm{rev}} .
$$

Because $\alpha^{2}-\alpha^{2}$ is a positive integer, the condition to observe the first revival requires that

$$
T_{\mathrm{rev}}=\frac{4 m L^{2}}{\pi \hbar} .
$$

This condition is of general validity regardless of the initial state $\psi_{0}(x)$ and its spectral decomposition. Accordingly, whenever $t=\nu T_{\text {rev }}$, with $\nu=1,2, \ldots$, the probability density undergoes a full revival, which implies that the signal $\psi(x, t)$ looks the same as $\psi_{0}(x)$ in amplitude, but is affected by a global phase factor $2 \pi \nu$. Nonetheless, it is worth noting that, when the $2 \pi \nu$ constraint is relaxed to $\pi(2 \nu-1)$, the mirror-symmetric (with respect to $x=0$ ) replica of the initial state (discussed below) is observed, which cannot be considered as a proper recurrence. Furthermore, if the input signal has a definite even or odd symmetry (i.e., it consists of a superposition of only even or odd eigenfunctions, respectively), revivals actually take place in shorter times, which does not invalidate the above condition. Note that in both cases the factor $\alpha^{2}-\alpha^{2}$ in Eq. (7) is proportional to 4. Indeed, because now all spectral components require the parity of the signal to be in phase, the constraint to $2 \pi \nu$ can be relaxed to simply $\pi \nu$. Hence, the observation of revivals 
occurs at an eighth of the general revival time $T_{\text {rev }}$. We define this new timescale as

$$
\tau=\frac{T_{\mathrm{rev}}}{8}=\frac{m L^{2}}{2 \pi \hbar}
$$

which will be used from now on as the reference time.

The presence of revivals of the initial state as well as recurrences at fractional times gives rise to a pattern with space and time symmetries referred to as a quantum carpet [4]. Typically the concept of quantum carpet is associated with the probability density, although similar symmetries can also be found in the amplitude and phase of the signal, or the quantum flux, which becomes more apparent when it is analyzed in terms of the associated streamlines or Bohmian trajectories 60. In order to determine the effects of decoherence on the flow of probability inside the cavity, here we proceed similarly, i.e., considering the supplementary aid of a Bohmian-type description. In brief, this formulation readily arises after recasting the signal in polar form [55], which provides us with a nonlinear transformation from a complex field to two real-valued ones, namely, the probability density $\rho$ and the phase $S$,

$$
\psi(x, t)=\rho^{1 / 2}(x, t) e^{i S(x, t) / \hbar} .
$$

It is well known 62] that, by virtue of the continuity equation for the probability density, it is possible to establish a relation between the latter and its flux through an underlying velocity field. In one dimension, this reads

$$
\begin{aligned}
J(x, t) & =\frac{\hbar}{2 m i}\left[\psi^{*}(x, t) \partial_{x} \psi(x, t)-\psi(x, t) \partial_{x} \psi^{*}(x, t)\right] \\
& =v(x, t) \rho(x, t)
\end{aligned}
$$

where the shorthand notation $\partial_{x} \equiv \partial / \partial x$ is used for convenience. Following this relation, now we have an unambiguous mechanism to monitor at a local level, i.e., at each point, how the probability density contracts and expands by interference as it flows across it back and forth inside the cavity. More specifically, these variations take place in compliance with the underlying local variations of the velocity field $v(x, t)$, which depend in turn on the local variations undergone by the phase field:

$$
v(x, t)=\frac{J(x, t)}{\rho(x, t)}=\frac{\partial_{x} S(x, t)}{m} .
$$

Of course, if there is a local velocity field, it is straightforward that, given any position, a trajectory can be obtained by integrating this coordinate-dependent and time-varying field in time, i.e., by integrating the equation of motion

$$
\begin{aligned}
\dot{x} & =v(x, t) \\
& =\frac{\hbar}{2 m i}\left[\frac{\psi^{*}(x, t) \partial_{x} \psi(x, t)-\psi(x, t) \partial_{x} \psi^{*}(x, t)}{\psi^{*}(x, t) \psi(x, t)}\right]
\end{aligned}
$$

These trajectories are the so-called Bohmian trajectories in the literature 63] and correspond to streamlines along which probability flows. Here they will provide us with a better idea of how the gradual suppressing effects led by decoherence are going to affect the degradation of quantum carpets, since Eq. (13) contains relevant information about the coherences. This is readily seen by substituting Eq. (4) into (13), which gives the analytical functional form

$$
\dot{x}=\frac{\hbar}{m}\left\{\frac{\sum_{\alpha^{\prime}>\alpha}\left|c_{\alpha}\right|\left|c_{\alpha^{\prime}}\right|\left[\varphi_{\alpha^{\prime}}(x) \partial_{x} \varphi_{\alpha}(x)-\varphi_{\alpha}(x) \partial_{x} \varphi_{\alpha^{\prime}}(x)\right] \sin \left(\omega_{\alpha \alpha^{\prime}} t-\delta_{\alpha \alpha^{\prime}}\right)}{\sum_{\alpha}\left|c_{\alpha}\right|^{2} \varphi_{\alpha}^{2}(x)+\sum_{\alpha^{\prime}>\alpha}\left|c_{\alpha}\right|\left|c_{\alpha^{\prime}}\right| \varphi_{\alpha}(x) \varphi_{\alpha^{\prime}}(x) \cos \left(\omega_{\alpha \alpha^{\prime}} t-\delta_{\alpha \alpha^{\prime}}\right)}\right\}
$$

which is very convenient both at a numerical level and also in order to determine the effective action of decoherence (discussed below in Sec. III).

Note that the same procedure can also be straightforwardly applied to light carpets inside optical fibers, resonant cavities, or multimode couplers 64. In such a case, the modes correspond to electromagnetic modes in compliance with the solutions to the Helmholtz equation for a cavity and the flux is determined by the Poynting vector (the flux of electromagnetic energy). Actually, if the Helmholtz equation is in paraxial form, its solutions will be formally equivalent (isomorphic) to those of the Schrödinger equation, except for the replacement of time by the longitudinal coordinate 65$]$.

\section{B. Symmetric and asymmetric carpets}

If the lowest (fundamental) mode transported by an optical fiber is pumped into a wider cavity, a multimode interference device [57, the light distribution inside the new space will exhibit carpet-type traits 47, since such a mode can now be described as a coherent superposition of proper modes of the cavity. To recreate this kind of behavior in a quantum context, here we consider single half-cosine amplitudes and a coherent superposition of them as initial Ansätze. The cavity is simulated by means of a one-dimensional infinite well with a width $L=50$ (arbitrary units), centered around $x=0$, while the width 
of the half-cosine input amplitude is $w=10$. The general expression for spectral decomposition of the input signal which will be considered is of the type

$$
\psi_{0}(x)=\sum_{\text {even } \alpha} c_{\alpha}^{e} \varphi_{\alpha}^{e}(x)+\sum_{\text {odd } \alpha} c_{\alpha}^{o} \varphi_{\alpha}^{o}(x),
$$

where $\varphi_{\alpha}^{e}$ and $\varphi_{\alpha}^{o}$ are as specified by (3).

In the case of a single half-cosine centered at $x=x_{0}$, the initial amplitude is given by

$$
\psi_{0}(x)=\left\{\begin{array}{cc}
\sqrt{\frac{2}{w}} \cos \left[\frac{\pi\left(x-x_{0}\right)}{w}\right], & \left|x-x_{0}\right| \leq \frac{w}{2}, \\
0, & \text { otherwise }
\end{array}\right.
$$

When recast in the form (15), the coefficients $c_{\alpha}$ read

$$
c_{\alpha}^{e}= \begin{cases}\frac{4}{\sqrt{w L}}\left(\frac{k_{0}}{k_{0}^{2}-k_{\alpha}^{2}}\right) \cos \left(k_{\alpha} x_{0}\right) \cos \left(\frac{k_{\alpha} w}{2}\right), & k_{\alpha} \neq k_{0}, \\ \sqrt{\frac{w}{L}} \cos \left(k_{0} x_{0}\right) & k_{\alpha}=k_{0},\end{cases}
$$

with $\alpha=2 n-1$, and

$$
c_{\alpha}^{o}= \begin{cases}\frac{4}{\sqrt{w L}}\left(\frac{k_{0}}{k_{0}^{2}-k_{\alpha}^{2}}\right) \sin \left(k_{\alpha} x_{0}\right) \cos \left(\frac{k_{\alpha} w}{2}\right), & k_{\alpha} \neq k_{0}, \\ \sqrt{\frac{w}{L}} \sin \left(k_{0} x_{0}\right) & k_{\alpha}=k_{0},\end{cases}
$$

with $\alpha=2 n$. In both cases, $k_{\alpha}=\alpha \pi / L$ and $k_{0}=\pi / w$ (notice that the condition $k_{\alpha}=k_{0}$ simply means that $w$ is an integer, even or odd, fraction of $L$ ). The quantum carpets generated by initial amplitudes centered at $x_{0}=0$ and 20 are displayed in Figs. 1(a) and (c), respectively. As it can be noticed, the loss of symmetry in the second case results in the revival time being reached at $t=T_{\mathrm{rev}}=8 \tau$ instead of at $t=\tau$, which is the case in the symmetric configuration. Nonetheless, in both cases it is possible to observe the presence of fractional recurrences, which is used in multimode interference devices (when dealing with light) to produce a number of identical copies of the same input state.

In order to elucidate how the probability density evolves inside the cavity, i.e., how the interference maxima that generate the recurrences arise, a number of Bohmian trajectories have been considered, evenly distributing their initial positions along the extension of the input signal. It can be seen how the initial diffraction launches the trajectories in a relatively fast manner towards the boundaries of the cavity, thus covering the whole available space inside it. Although the appearance of recurrences and revivals is independent of the input signal, the initial boost strongly depends on it, as shown elsewhere 60]. The same behavior can be observed in both symmetric and asymmetric cases, though with the difference that in the latter case trajectories on one side (a)

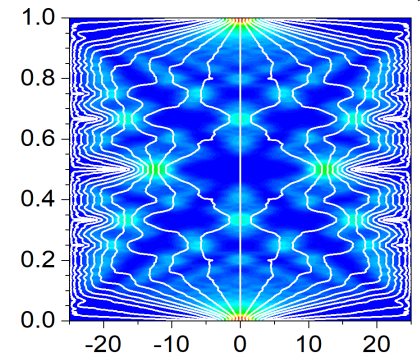

(c)

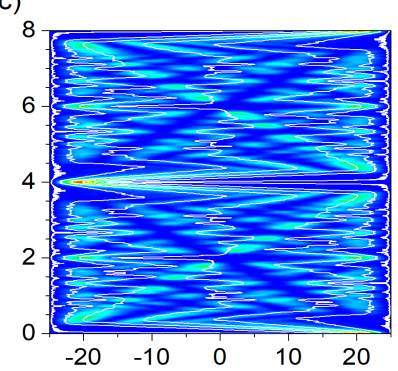

(e)

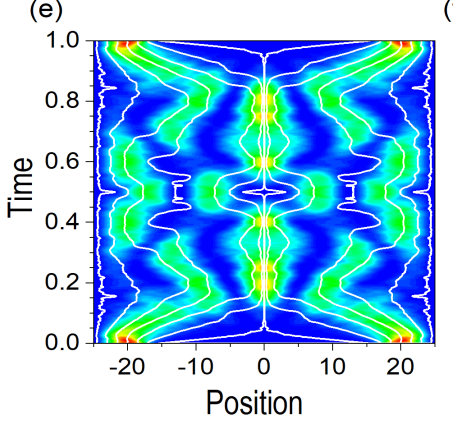

(b)

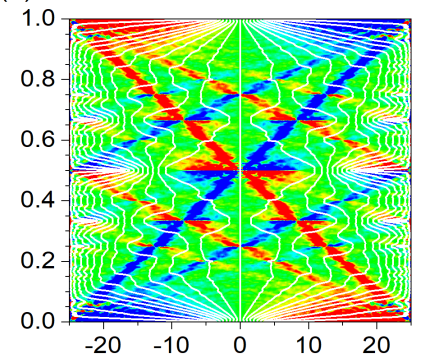

(d)

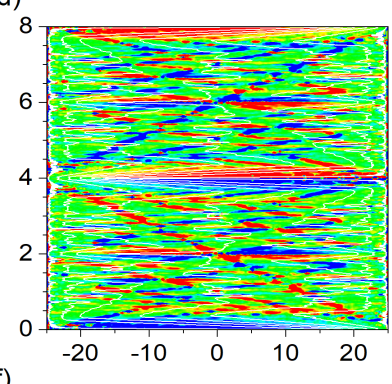

1.0

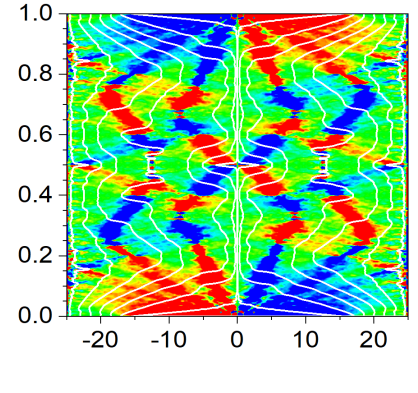

FIG. 1. Quantum carpets generated by half-cosine-type amplitudes: probability density (left column) and velocity field (right column). In all cases, $L=50, w=10, m=1$ and $\hbar=1$ (all quantities are given in arbitrary units). Time is measured in units of $\tau$, as defined in Eq. (9). From top to bottom, single half-cosine input amplitudes centered at $x_{0}=0$ (upper row) and $x_{0}=20$ (middle row), and coherent superposition of two of them centered at $x_{0}= \pm 20$ (lower row). In the color scale for the density plots, blue is used to denote the lowest values (zero values for the probability density and maximum negative values for the velocity field) and red the highest values (maximum positive values for the velocity field). To better specify the flow inside the cavity, a number of Bohmian trajectories (white solid lines) have been included in each panel.

of the input signal have to travel a larger distance than those started on the other side. Nonetheless, because of this asymmetry, the trajectories on the right side of $\psi_{0}$ gradually start being launched towards the left side of the cavity, until at $t=T_{\text {rev }} / 2$ they all gather and produce a full revival of the input signal at $x=-x_{0}=-20$. This intricate motion can be better understood by analyzing the carpets associated with the velocity field, in Figs. 1(b) and (d), respectively for each case. As it can be noticed, the local velocity values reach very sudden changes (red and blue regions), which act on the trajectories in the same way as bumpers and other targets in 
a pinball machine.

So far, the presence of a single input signal only affects the interference process that follows the diffraction of such a signal. Another case of interest is that of two coherent input signals, analogous to a two-slit experiment carried out inside the box. In this regard, we consider the above asymmetric input and construct a superposition with its mirror image, i.e., we now consider an input signal of the type

$$
\Psi_{0}(x)=\left\{\begin{array}{cc}
\sqrt{\frac{1}{w}} \cos \left[\frac{\pi\left(x \pm x_{0}\right)}{w}\right], & \left|x \pm x_{0}\right| \leq \frac{w}{2} \\
0, & \text { otherwise }
\end{array}\right.
$$

with $x_{0}=20$. Because of its even symmetry with respect to $x=0$, all odd-symmetric terms in 15 vanish $\left(c_{\alpha}^{o}=\right.$ $0)$

$$
c_{\alpha}^{e}= \begin{cases}4 \sqrt{\frac{2}{w L}}\left(\frac{k_{0}}{k_{0}^{2}-k_{\alpha}^{2}}\right) \cos \left(k_{\alpha} x_{0}\right) \cos \left(\frac{k_{\alpha} w}{2}\right), & k_{\alpha} \neq k_{0}, \\ \sqrt{\frac{2 w}{L}} \cos \left(k_{0} x_{0}\right), & k_{\alpha}=k_{0},\end{cases}
$$

with $\alpha=2 n-1$. The corresponding carpets for the probability density and the velocity field are displayed in Figs. 1(e) and (f), respectively. As it can readily be noticed, the presence of the second signal reduces the revival time for the probability density to $\tau$, which does not depend on the particular choice of $x_{0}$ but on the equal weight assigned to both signals. Again, the pinball-type structure displayed by the velocity field becomes apparent, with the addition of a sort of channeling pattern, which is a trait of an incipient Young-type interference substructure [66], typical of two wave-packet superpositions [55, 67.

\section{DECOHERENCE-INDUCED DYNAMICS}

\section{A. Effective modeling of decoherence}

Decoherence arises from the interaction of a quantum system with a surrounding environment and manifests in the gradual loss of coherence, i.e., in its ability to display interference [6, 7]. Formally, the different coupling of each system proper state with environmental states eventually leads to the suppression of their interference due to the orthogonality of the latter states 68. This is readily understood by inspecting its manifestation in the corresponding density matrix elements. Taking into account the states here, these elements are going to be of the form

$$
\begin{aligned}
\rho\left(x, x^{\prime} ; t\right)= & \psi^{*}\left(x^{\prime}, t\right) \psi(x, t) \\
= & \sum_{\alpha^{\prime}, \alpha} c_{\alpha} c_{\alpha^{\prime}}^{*} \varphi_{\alpha}(x) \varphi_{\alpha^{\prime}}\left(x^{\prime}\right) \\
& \quad \times e^{-i\left(E_{\alpha}-E_{\alpha^{\prime}}\right) t / \hbar} \mathcal{D}_{\alpha \alpha^{\prime}}\left(x, x^{\prime} ; t\right),
\end{aligned}
$$

where $\mathcal{D}_{\alpha \alpha^{\prime}}\left(x, x^{\prime} ; t\right)$ accounts for the coherence loss term, which is going to act on the off-diagonal elements of the density matrix. Following models considered in the literature to describe two-state decoherence [68, 69] as well as more detailed numerical models [50, 70, 71, clearly this term should annihilate any correlation between two different energy states $\alpha$ and $\alpha^{\prime}$ as well as between two different spatial points $\left(x, x^{\prime}\right)$. Furthermore, this coherence damping should show an exponentially decreasing , behavior with time. Accordingly, we have considered a simple model for this dissipating term

where

$$
\mathcal{D}_{\alpha \alpha^{\prime}}\left(x, x^{\prime} ; t\right)=e^{-\beta_{\alpha \alpha^{\prime}} t-\Lambda\left(x-x^{\prime}\right)^{2} t},
$$

$$
\beta_{\alpha \alpha^{\prime}} \equiv \gamma \omega_{\alpha \alpha^{\prime}}
$$

accounts for the gradual suppression of the coherences between different modes, with $\gamma$ a coefficient used for control (to speed up or to slow down the decoherence rate, constant for all combinations of modes). Note that since $\omega_{\alpha \alpha^{\prime}}$ is positive [according to the definition (6)], $\beta_{\alpha \alpha^{\prime}}$ is also positive, thus ensuring a gradual damping with time. On the other hand, the factor

$$
\Lambda=\frac{2 \pi \hbar}{m L^{3}}
$$

is the usual localization rate 2, 69, which determines the how fast two points of the signal, at $x$ and $x^{\prime}$, lose mutual coherence with time. The particular choice here produces an overall decay going like $1 / L$ for small distances $\left|x-x^{\prime}\right|$ (the energy term is the dominant one) and like $L$ for large ones (the spatial term becomes the leading one, as required to annihilate the prevalence of coherence along the secondary diagonal).

By inspecting (21), we notice that the spatial part seems to play a minor role when we look at the probability density, since this quantity only takes into account the diagonal elements

$$
\rho(x ; t)=\sum_{\alpha}\left|c_{\alpha}\right|^{2} \varphi_{\alpha}^{2}(x)+\sum_{\alpha^{\prime}>\alpha}\left|c_{\alpha} \| c_{\alpha^{\prime}}\right| \varphi_{\alpha}(x) \varphi_{\alpha^{\prime}}(x) \cos \left(\omega_{\alpha \alpha^{\prime}} t-\delta_{\alpha \alpha^{\prime}}\right) e^{-\beta_{\alpha \alpha^{\prime}} t},
$$

which do not include nonlocal correlations but mutual coherence between energy proper states, responsible for the appearance of the carpets. Following the prescription provided in [51, the corresponding Bohmian-type trajectories 
are given by the expression

$$
\dot{x}=\frac{\hbar}{m}\left\{\frac{\sum_{\alpha^{\prime}>\alpha}\left|c_{\alpha}\right|\left|c_{\alpha^{\prime}}\right|\left[\varphi_{\alpha^{\prime}}(x) \partial_{x} \varphi_{\alpha}(x)-\varphi_{\alpha}(x) \partial_{x} \varphi_{\alpha^{\prime}}(x)\right] \sin \left(\omega_{\alpha \alpha^{\prime}} t-\delta_{\alpha \alpha^{\prime}}\right) e^{-\beta_{\alpha \alpha^{\prime}} t}}{\sum_{\alpha}\left|c_{\alpha}\right|^{2} \varphi_{\alpha}^{2}(x)+\sum_{\alpha^{\prime}>\alpha}\left|c_{\alpha}\right|\left|c_{\alpha^{\prime}}\right| \varphi_{\alpha}(x) \varphi_{\alpha^{\prime}}(x) \cos \left(\omega_{\alpha \alpha^{\prime}} t-\delta_{\alpha \alpha^{\prime}}\right) e^{-\beta_{\alpha \alpha^{\prime}} t}}\right\},
$$

which does not include any term depending on the spatial correlations, because the evaluation is locally performed in contrast to two-slit scenarios, where the coherence loss between spatially distinct states has to gradually vanish [51. In spite of the apparently complex functional form displayed by Eq. (26), the asymptotic behavior of the trajectories can easily be inferred in the cases of strong and weak decoherence. Thus, for a strong decoherence (in terms of $\gamma$ ), in the leading term in the denominator of Eq. (26) is essentially dominated by the time-independent population term, a rather weak timedependent contribution still persists in the numerator (in terms of the oscillatory functions, apart from the decaying factors). This contribution is enough to make the trajectories approach their stationary state condition relatively fast. This stationarity condition corresponds to the population distribution (discussed below). On the other hand, for a weak decoherence, the rich structure displayed by trajectories (see Fig. 1) will get smoother until they completely stop and remain steady. In either case, the trajectories will tend to distribute according to the bare sum of partial distributions, the long-time limit of 25 , i.e.,

$$
\rho_{\infty}(x)=\sum_{\alpha}\left|c_{\alpha}\right|^{2} \varphi_{\alpha}^{2}(x)
$$

Accordingly, because there is no energy dissipation, there will not be spatial localization either, which means that the trajectories will not remain within the region covered by the input state. On the contrary, they will distribute according (27) and satisfying the so-called noncrossing rule, i.e., trajectories cannot cross one another, because nonlocal spatial information still remains 52,53 .

\section{B. Decoherence in the position representation}

Taking into account the above-described effective model, let us know analyze the pattern erasure undergone by the quantum carpets discussed in Sec. [IB in the presence of decoherence. To this end, for convenience but without any loss of generality, from now on we consider $\gamma=2 / 5 \pi$. With this value for the friction it is ensured that, at $t=\tau$, all energy-dependent decoherence factors amount to $\left(\alpha^{\prime 2}-\alpha^{2}\right) / 10$, thus giving rise to the smoothing of the carpets in all cases in about four times $\tau$; of course, some terms will decay faster than others depending on the difference $\left(\alpha^{\prime 2}-\alpha^{2}\right) / 10$. Results for the three cases displayed in Fig. 1 are shown in the corresponding panels of Fig. 2. As can be seen, the long-time limit in all (a)

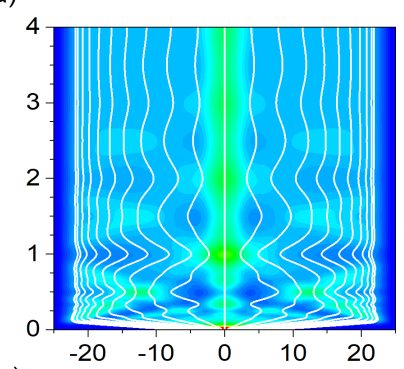

(c)

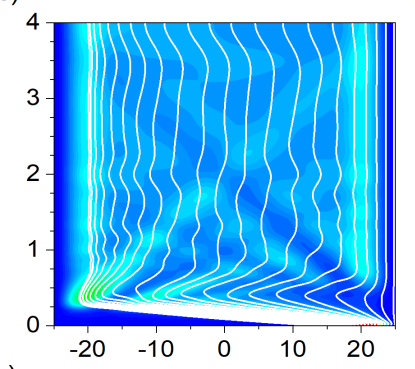

(e)

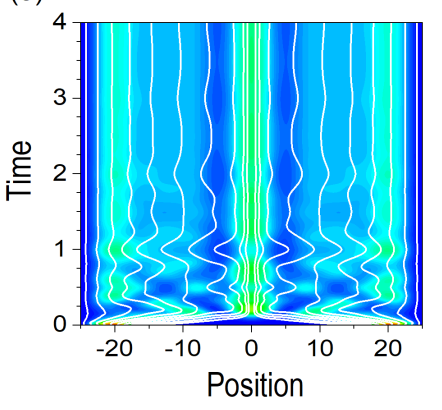

(b)

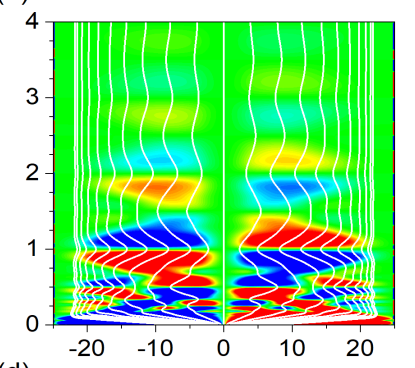

(d)

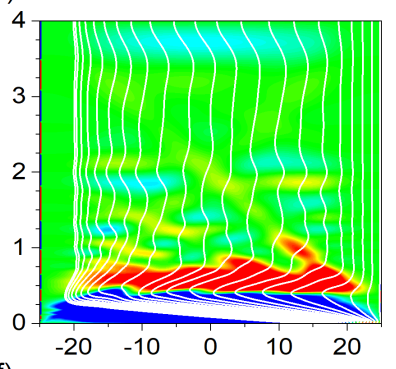

(f)

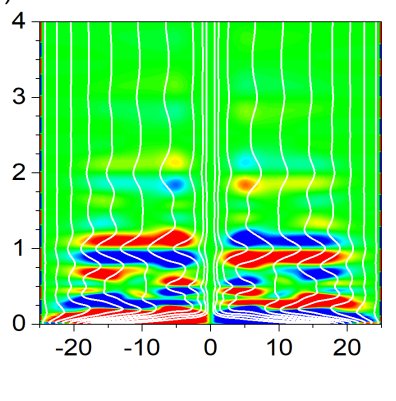

FIG. 2. Same as in Fig. 1 but with the presence of decoherence, with $\gamma=2 / 5 \pi$. To better show the coherence loss effects, in all cases a total propagation up to four times the recurrence period of the symmetric case $(4 \tau)$ has been chosen. All quantities are given in arbitrary units.

cases consists of a series of steady trajectories, unevenly but symmetrically distributed with respect to the center of the cavity, although one might intuitively expect a relative localization around a certain position, in particular, the center of the input distribution. This is in compliance, though, with the delocalization also exhibited by the probability density. Note that in Fig. 2(a), although a prominent central maximum becomes apparent as time proceeds, we also observe that the probability distribution partly spreads out towards the borders of the cavity. This behavior finds a clear explanation when we look at the velocity field, shown in Fig. 2(b), which gradually loses its pinball-like structure and now 

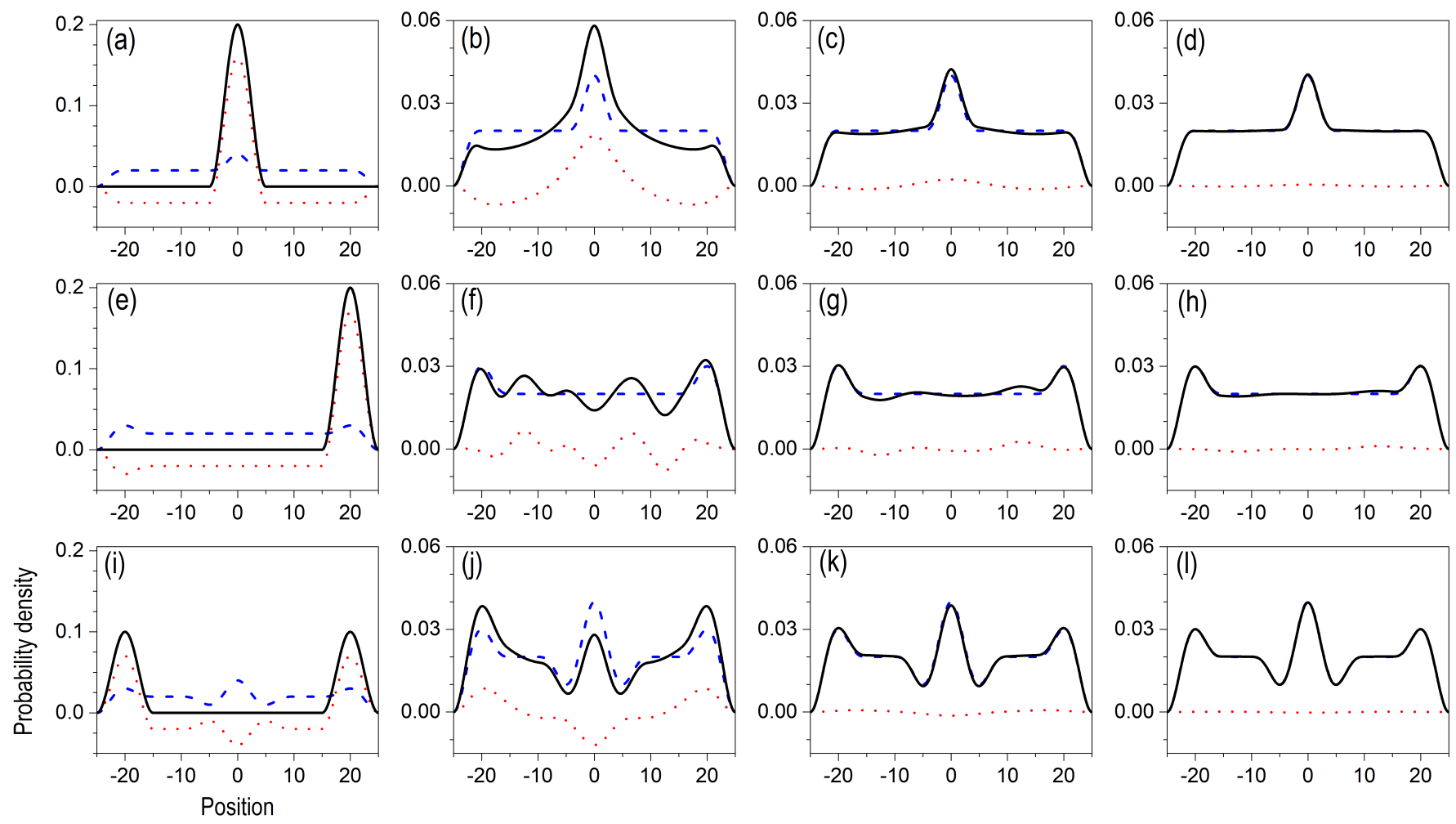

FIG. 3. Snapshots of constant-time profiles for the three input states here considered (see Sec. II B). From top to bottom: center-symmetric half-cosine $\left(x_{0}=0\right)$, asymmetric half-cosine $\left(x_{0}=20\right)$ and superposition of two half-cosines (with $\left.x_{0}=20\right)$. From left to right: $t=0, t=\tau, t=3 \tau$ and $t=5 \tau$. In each panel, the probability density is displayed with a black solid line, while the distributions related to the populations and the coherences are denoted by the blue dashed line and red dotted line, respectively. In all cases $L=50, w=10, m=1=\hbar$ and $N=50$. All quantities are given in arbitrary units.

presents an alternating distribution of positive and negative regions. As a consequence of this structure, the trajectories are smoothly driven until they completely stop instead of undergoing sudden and fast motions. To some extent, this situation is reminiscent of the calm waters that come after the rapids in a river, which occurs when turbulence sources disappear. In our context, this happens because the influence of the frequencies involved in the interference process, which originates in nodal regions (vortices) and hence strong local variations in the velocity field, is suppressed. The same trend is observed in Fig. 2(c). Although the configuration is asymmetric, it can also be seen that the trajectories smoothly move towards the symmetric position (with respect to $x=0$ ) and then, some of them, backward again, distributing nearly homogeneously across the cavity. This is in compliance with the behavior shown by the velocity field in Fig. 2(d). Finally, the case of the superposition, shown in Fig. 2(e), looks pretty similar to that shown in Fig. 2(a) for the symmetric single half-cosine, although there are two additional accumulation regions at $x \approx \pm 20$, apart from the central one.

To better understand the behavior exhibited by both the probability density and the trajectories in Fig. 2 , let us now analyze the time evolution of the probability density, separating for our purpose the contributions coming from populations and coherences. Accordingly, some snapshots of the three quantities are displayed in Fig. 3 for $t=0, \tau, 3 \tau$, and $5 \tau$ (from left to right) for the three cases here considered (from top to bottom). As it can be noticed, at $t=0$ the probability density (black solid line) corresponds to either single localized peaks or two peaks, depending on whether we have a single half-cosine input signal or a superposition of two of them, respectively. Now, if we inspect more closely these distributions, separating the contributions coming from their populations (blue dashed line) and their coherences (red dotted line), some interesting features readily emerge. First of all, note that, as expected, the populations contribution is stationary, which is due to the fact that this is a dissipation-free model, where thermalization effects that reorganize populations in each cavity mode are disregarded. As it was mentioned above, it is a purely decoherence model, which only influences the state-state correlations. Accordingly, although the initial probability density is peaked at some particular place or places, the contribution associated with the populations exhibits a certain degree of delocalization across the cav- 

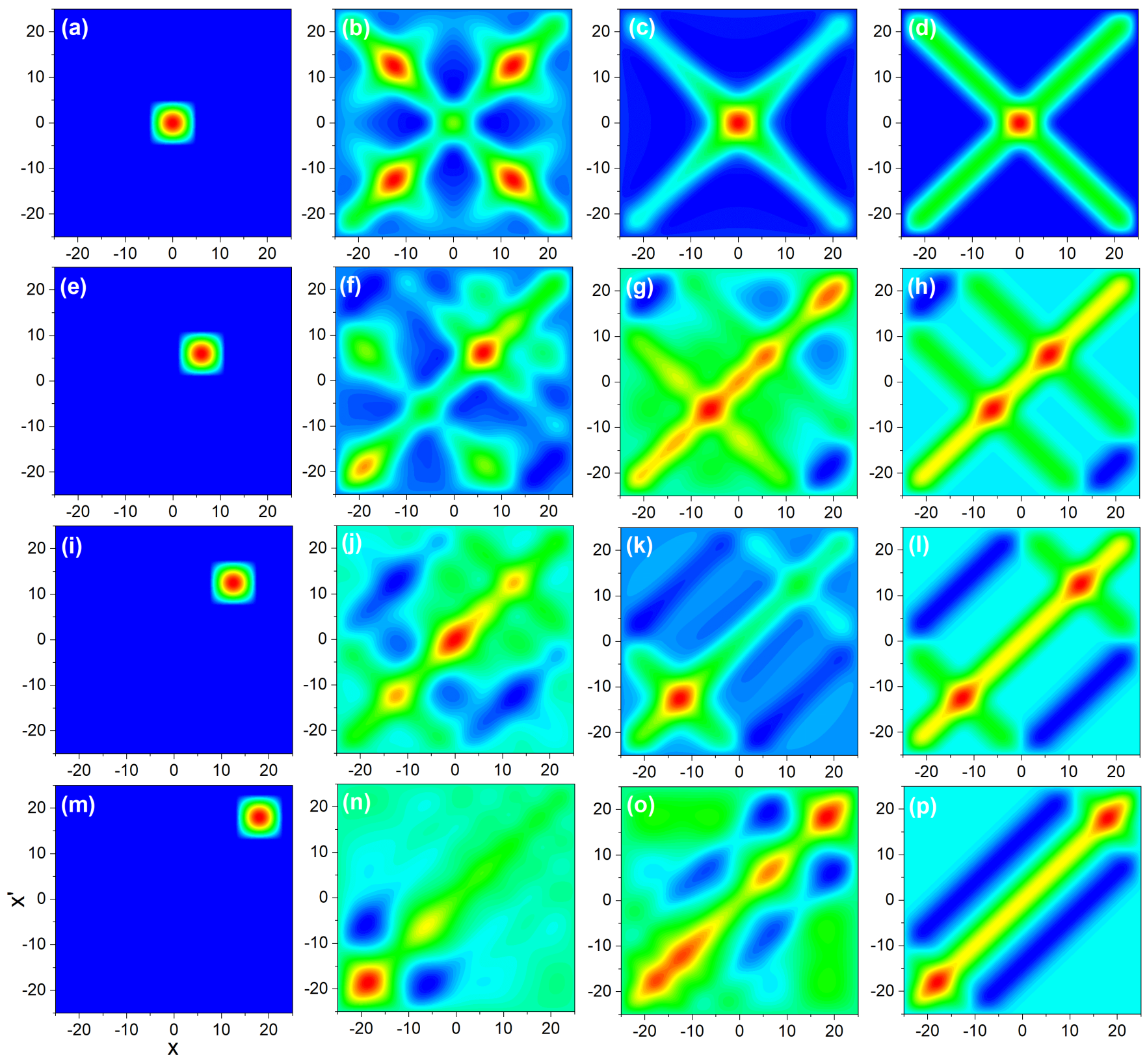

FIG. 4. Real part of the density matrix for a single half-cosine initial input with $\Lambda=0$. From top to bottom rows: $x_{0}=0$, $x_{0}=6, x_{0}=12.5$ and $x_{0}=18$. From left to right: $t=0, t=\tau / 2, t=\tau$ and $t=20 \tau$. As before, in all cases $L=50, w=10$, $m=1=\hbar$ and $\gamma=2 / 5 \pi$. All quantities are given in arbitrary units.

ity. This is going to the asymptotic distribution once coherence is totally suppressed (see panels in the last column). Depending on whether a symmetric or an asymmetric input signal is considered, we observe the presence or absence of a maximum at $x=0$, respectively. This maximum plays the role of a certain effective barrier in the symmetric configurations: Trajectories started on either side will never be able to cross to other side [60, 72]. To some extent, the dynamics on either side of the central maximum is going to be ruled by this maximum and the borders of the cavity, in a fashion similar to an effective two-wave superposition (although with a more com- plex interference process in between, as shown in Fig. 1, top and bottom panels). The same is not observed in the asymmetric case, because the absence of the central maximum allows the trajectories to move from one side of the cavity to the other and vice versa, since now these dynamics are ruled by the two marginal maxima, i.e., the one corresponding to the input signal and its mirror image. All these dynamics, on the other hand, are directly mediated by the changes in time undergone by the coherence terms (red dotted line), i.e., the complex interference processes generated by the correlations established among all modes. When the decoherence damping 

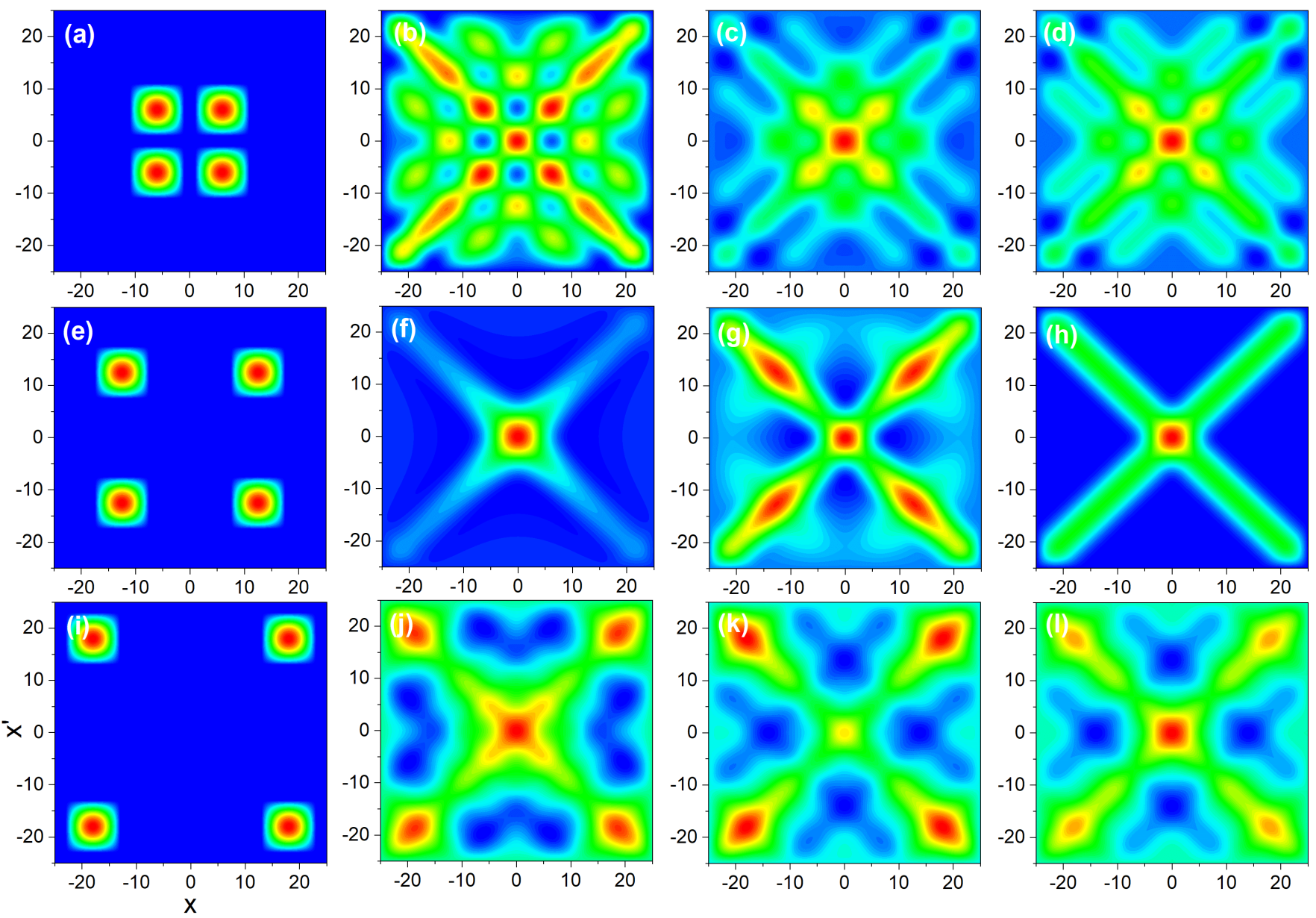

FIG. 5. Real part of the density matrix for a coherent superposition of two half-cosine initial inputs with $\Lambda=0$. From top to bottom rows: $x_{0}=6, x_{0}=12.5$ and $x_{0}=18$. From left to right: $t=0, t=\tau / 2, t=\tau$ and $t=20 \tau$. As before, in all cases $L=50, w=10, m=1=\hbar$ and $\gamma=2 / 5 \pi$. All quantities are given in arbitrary units.

factor starts acting on them, they gradually disappear, which removes the oscillatory behaviors displayed with time and lets the so-far "screened" population contribution emerge, as it can be seen in the last two columns for all cases. Accordingly, once the oscillatory (timedependent) term has been canceled out, the trajectories are also going to stop their wandering motion inside the cavity, remaining stationary, as seen in Fig. 2.

So far we have focused on the probability density, trying to understand its dynamics in terms of the underlying velocity field and the corresponding Bohmian trajectories. Let us now consider the more general view provided by the corresponding density matrix, firs assuming a vanishing localization rate, i.e., $\Lambda=0$, but keeping finite the value of $\gamma$ (as before, $\gamma=2 / 5 \pi$ ). When proceeding this way for a single input signal, we find that, as time proceeds, a rather structured pattern arises independently of the value of $x_{0}$, as shown in Fig. 4 Each column shows a snapshot of the real part of the density matrix for the single half-cosine initial Ansatz, namely, $t=0, \tau / 2, \tau$, and $20 \tau$, from left to right, and four different positions of the signal center, namely, $x_{0}=0, x_{0}=6, x_{0}=12.5$, and 18 , from top to bottom. Intuitively, one would expect that, with time, the density distributes along the diagonal, as it corresponds to the real part of the density operator, which what we are showing here. However, what we observe is a very symmetric structure, which looks the same asymptotically along the main diagonal and also on both sides with respect to the secondary diagonal (last column). This is because spatial correlations have not been properly removed, but only those in the energy domain. The same behavior is also observed in the case of the initial superposition, as it is shown in Fig. 5 for $x_{0}=6,12.5$, and 18 , from top to bottom, and the same four times, where a rather four-fold symmetric pattern emerges. Interestingly, the case for input signals at localized at the center of each half of the cavity $\left(x_{0}=12.5\right)$ actually becomes asymptotically equal to the pattern of a single input signal at the center of the cavity $x=0$. This coincidence arises from the time-symmetry displayed by these two patters, with the latter having a recurrence at $\tau / 2$ with the same form of the superposition. 

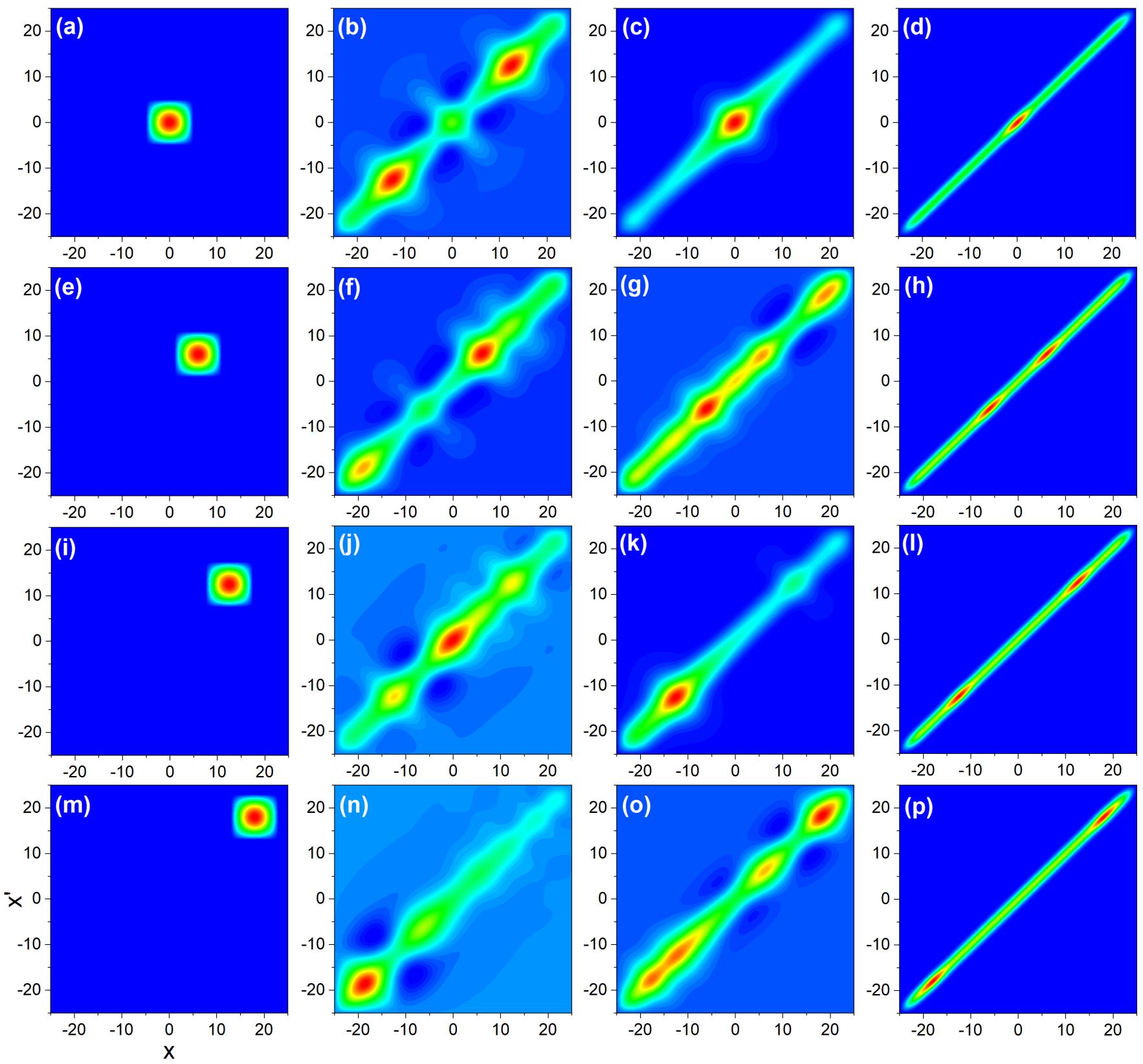

FIG. 6. Same as in Fig. 4 but with $\Lambda$ as given by Eq. 24.

To some extent, one might consider that the above results for $\Lambda=0$ are counter intuitive. In principle, one would expect a gradual suppression of all terms outside the main diagonal of the real part of the density matrix [50, 70, 71, 73]. However, although certainly the rich interference structure outside this diagonal disappears, certain traits related to the $x-x^{\prime}$ exchange symmetry still persist. This is the key aspect that makes necessary in the model here the presence of a space-dependent damping factor, i.e., a spatial localization rate, $\Lambda$. This term can be determined analytically 69] by solving the von Neumann equation for a free particle acted upon by a scattering-type environment. Here we have combined this localization rate with the one that is expected from the cancellation of the interference between different energy modes of the cavity. The behavior of the real part of the density matrix when this term is added can be seen in Figs. 6and 7, which are the respective counterparts of Figs. 4 and 5 for nonvanishing $\Lambda$, with its value given by (24). As it can be seen, now the model produces the suppression of both energy and space correlations, thus providing a full phenomenological description to the loss of coherence inside the cavity. In the particular case we are dealing with here, though, two-point space correlations do not directly affect the trajectories (quantum flux), but only the oscillations coming from interference between 

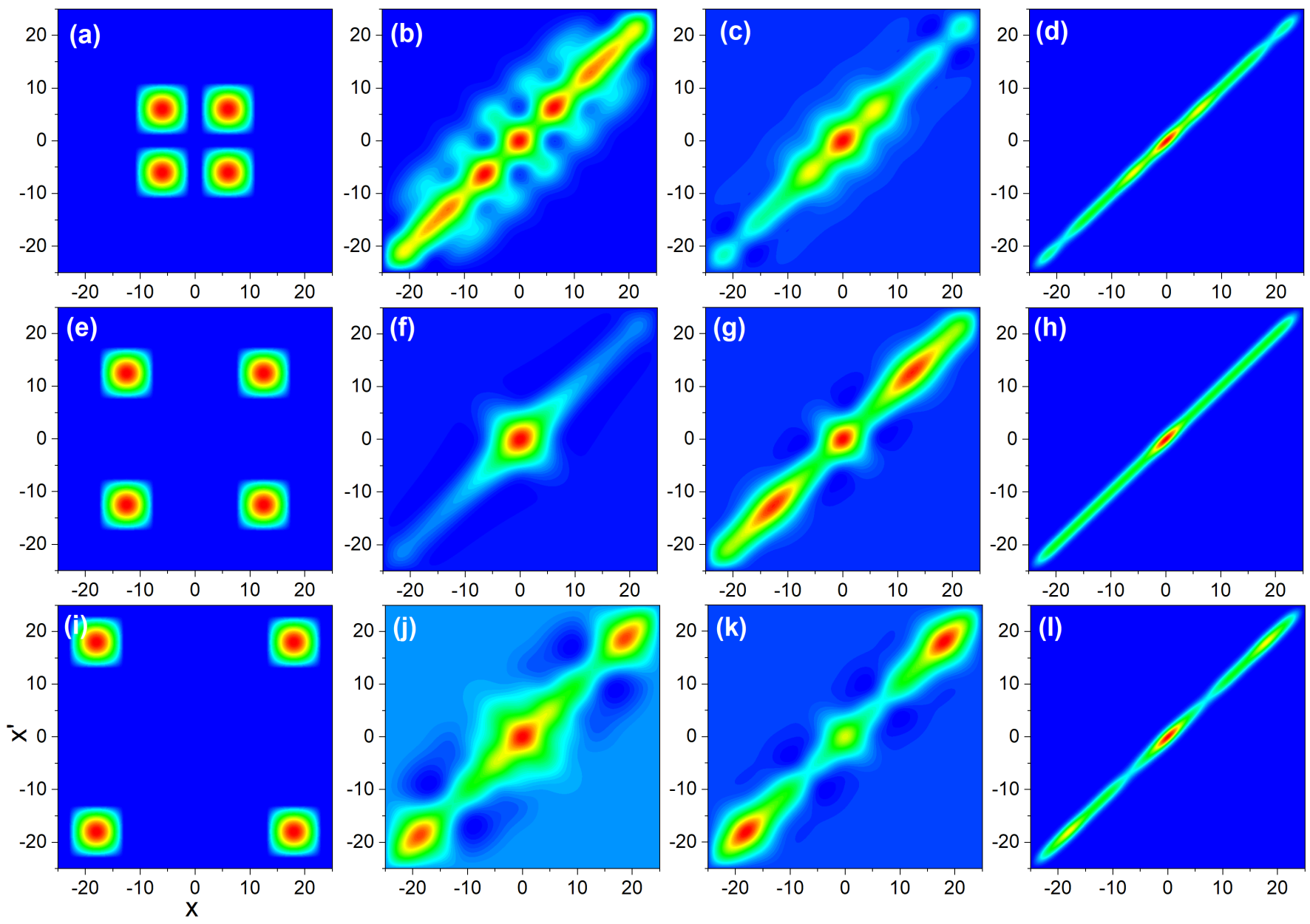

FIG. 7. As in Fig. 5 with $\Lambda$ as given by Eq. 24.

different energy modes. This is consistent with the fact that the localization rate does not appear at all in the equation of motion (26). Now, although this view seems more apparent in the case of the single input signals, in the case of the superposition it seems that such a term should play a role, as it is observed in two-slit-type studies where it has also been considered [51, 53. If this is not the case, it is precisely because of the spectral decomposition of the input signal that the equation of motion of the trajectories is based on, which neglects the fact of two spatially separate signals and reconsiders the initial Ansatz as a whole, unlike the two-slit scenario, where no energy decomposition is considered and therefore the equation of motion is fully based on what happens in the position space.

\section{Decoherence in the energy representation}

To further analyze the implications of the model, now we are going to analyze its consequences in the energy domain. To this end, we consider the purity [1], which here acquires the explicit functional form

$$
\begin{aligned}
\chi(t) & =\operatorname{Tr}\left(\rho^{2}\right) \\
& =\sum_{\alpha}\left|c_{\alpha}\right|^{4}+2 \sum_{\alpha^{\prime}>\alpha}\left|c_{\alpha}\right|^{2}\left|c_{\alpha^{\prime}}\right|^{2} e^{-2 \beta_{\alpha \alpha^{\prime}} t},
\end{aligned}
$$

with its long-time limit

$$
\chi_{\infty}=\sum_{\alpha}\left|c_{\alpha}\right|^{4}
$$

This quantity provides us with a reliable measurement of the degree of mixedness undergone by an initially pure quantum system and, therefore, of the effects induced by decoherence. In principle, the density in 28 refers to the reduced density, i.e., after tracing over the environmental degrees of freedom. Since the latter is included here in a phenomenological manner, the density makes direct reference to the density describing the carpet. Nonetheless, notice that the environmental effects appear explicitly in the form of the exponential damping factors.

The time-dependence of the purity for an interval equivalent to ten times $\tau$ is displayed in Fig. 8(a) for half-cosine input signals centered at different values of 

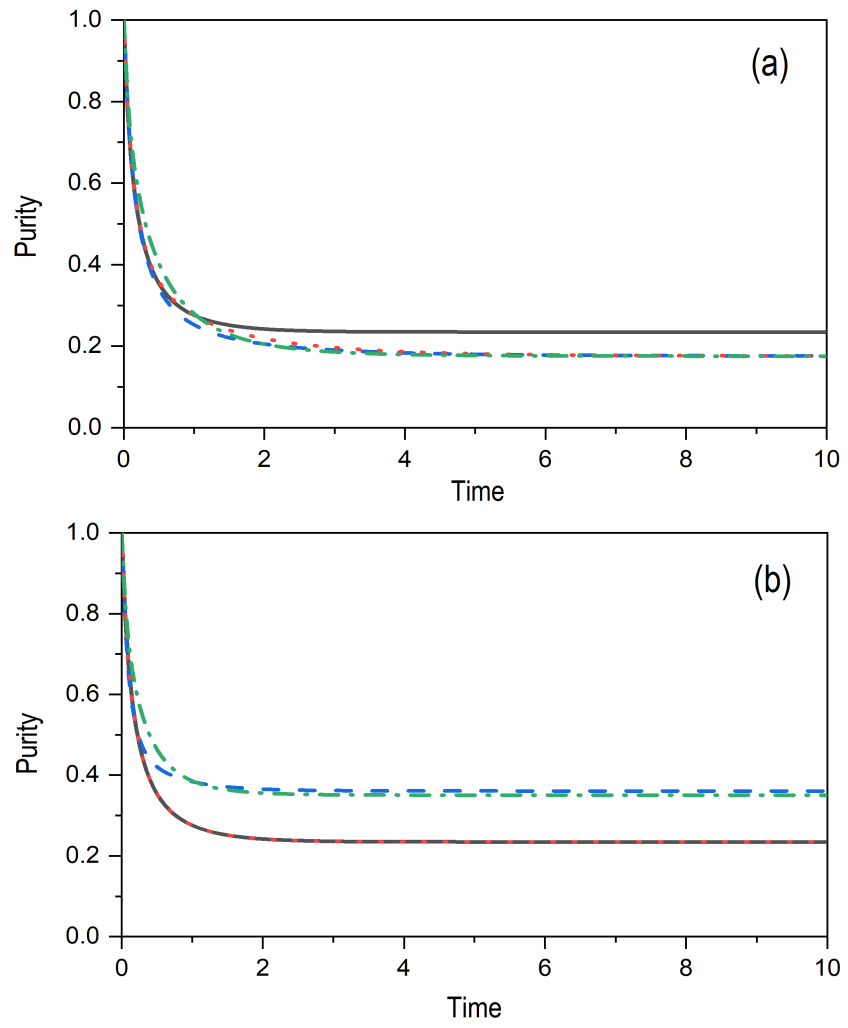

FIG. 8. Purity over a time $10 \tau$ for (a) single input signals and (b) their symmetric double signal counterparts. The centers of these inputs are $x_{0}=6$ (blue dashed line), $x_{0}=12.5$ (red dotted line) and $x_{0}=18$ (green dash-dotted line) and decrease by one to their corresponding double waves. To set a reference to compare with, the result for the input signal centered at $x_{0}=0$ is denoted by a black solid line in both panels. In all cases the parameters considered are $\hbar=1, m=1, L=50$, $w=10$ and $\gamma=2 / 5 \pi$. All quantities are given in arbitrary units.

$x_{0} \neq 0$. As it can be noticed, all these cases reach the same asymptotic value of nearly $\chi_{\infty} \lesssim 0.2$, below the reference value for $x_{0}=0, \chi_{\infty} \gtrsim 0.2$. However, the falloff slightly differs for each case, which is related to the fact that the decay factor depends on the frequencies (energy differences) involved as well as the number of modes and their weight contributing to the corresponding signal. A similar trend is also observed in the two-signal superpositions, shown in Fig. 8(b), with the initial state consisting of the half-cosines centered at the same $x_{0}$ values and the mirror image (with respect to $x=0$ ). In this case, though, the limiting value is $\chi_{\infty} \lesssim 0.4$, nearly twice the value for the single signals, except in the case $x_{0}=12.5$ (red dotted line), which is the same as for the reference case because of the time-symmetry reasons explained above.

In order to determine whether the $x_{0}=0$ case is an exception, we proceed to revise the asymptotic value 290 for the whole range between $x_{0}$ and 20 , which is the maximum value without truncating the shape of the input

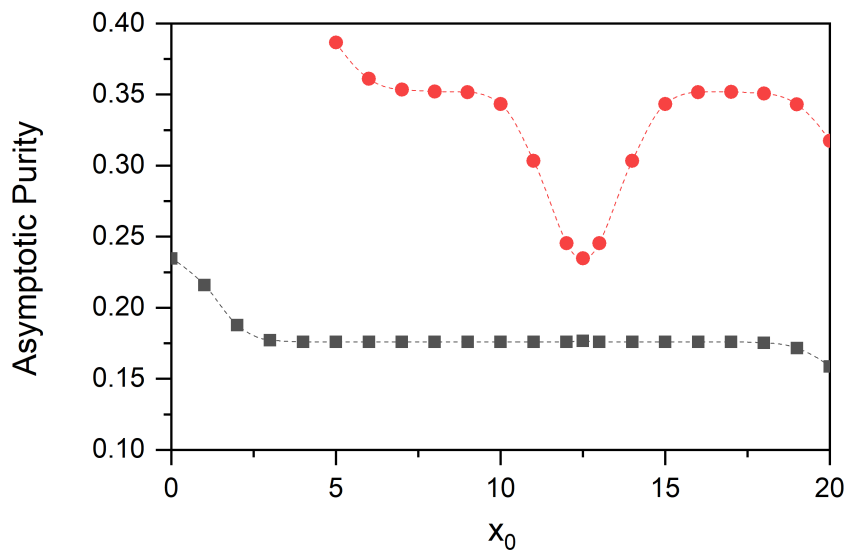

FIG. 9. Long-time (asymptotic) values of the purity as a function of $x_{0}$ for single (black squares) and double (red circles) input signals. For easier visualization, colored dotted lines joining the data have been added. In all cases, the parameters are $m=1, \hbar=1, L=50, w=10$ and $\gamma=2 / 5 \pi$. All quantities are given in arbitrary units.

signal (note that the cavity extends to $x=L / 2=25$ and the half-width of the input signal is $w=5$ ). We proceed the same way with the corresponding superpositions, although this time the limitation is extended also from below (the minimum $x_{0}$ is 5 ) in order to avoid the overlapping of the two signals. The results are shown in Fig. 9, where black squares denote the values considered for the single half-cosines, while the red circles represent those for the superpositions. As can be clearly seen, in both case there is a range where more or less all asymptotic values remain the same. Important deviations correspond to the extreme values of $x_{0}$, where the long-time purity value undergoes an increase for lower values of $x_{0}$ and decreases for the larger ones. On the other hand, a remarkable feature is the dip observed in the case of the superpositions, which explains the coincidence between the black solid line and the red dotted line of Fig. 8(b): Because the single half-cosine centered at $x_{0}=0$ and the two half-cosine superpositions for $x_{0}=L / 4=12.5$ are equivalent from the point of view of time symmetry, their purities must coincide, even in the long-time limit. This is precisely what we observe here if we compare the lowest value of the dip with the initial value for the single half-cosine (i.e., the one for $x_{0}=0$ ). It is thus clear that, in spite of the simplicity of the model, it can provide us with useful information about the role of symmetries in decoherence processes and hence a deeper understanding of the carpet-suppression dynamics.

Some additional information can still be extracted if we analyze the time scales involved in the decay of the purity. To that end, it is interesting to consider the decay time-scales related to $\left(\alpha, \alpha^{\prime}\right)$ pairs of modes, given by $1 / \beta_{\alpha \alpha^{\prime}}$. These decay times are independent of the particular input signal selected, since they do not include the contribution of the particular weight assigned to each pair. Figure 10(a) illustrates the distribution of decay 

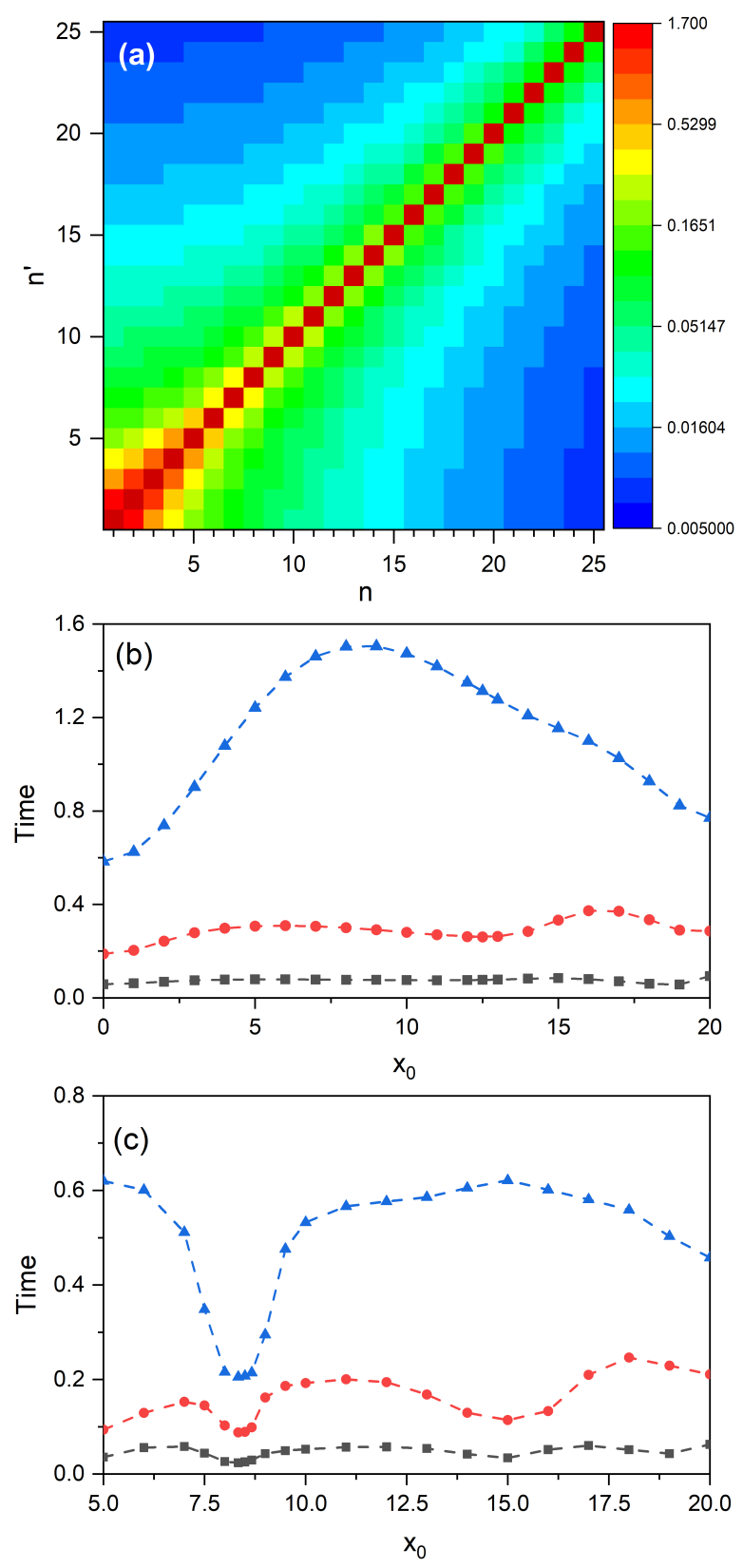

FIG. 10. (a) Color map showing the decay times associated with $\left(\alpha, \alpha^{\prime}\right)$-pairs of modes, where the transition from red to blue denotes shorter and shorter decay times (faster and faster decay). (b) Characteristic times $t_{1}$ (black squares), $t_{2}$ (red circles) and $t_{3}$ (blue triangles) for single input states as a function of the latter center $x_{0}$. (c) Same in (b) but for double input signal. For easier visualization, colored dotted lines joining the data have been added. In all cases, the parameters are $m=1, \hbar=1, L=50, w=10$ and $\gamma=2 / 5 \pi$. All quantities are given in arbitrary units.

times by means of a color map, where the transition from red to blue denotes shorter and shorter decay times, as expected for pairs of modes with bigger and bigger energy differences. Note that in the particular case of the diagonal (dark red color) the decay time becomes infi- nite because $\alpha^{\prime}=\alpha$, this being the main reason why the real part of the density matrix collapses asymptotically towards this diagonal in the energy representation. This map thus provides us with a general picture of decay times that does not depend on the particular choice of the input signal, as mentioned above.

It is clear that the choice of a given input signal is going to have some consequence on the decay of the carpet, because not only does the time scale $1 / \beta_{\alpha \alpha^{\prime}}$ rule its decoherence dynamics, but also the particular weight $\left|c_{\alpha}\right|\left|c_{\alpha^{\prime}}\right|$ is going to play a role depending on whether or not it is important in the superposition (with respect to other crossed terms or even the populations weights $\left|c_{\alpha}\right|^{2}$ ). To analyze these consequences, now we focus on the shape displayed by the purity and fit it with a three-time exponentially decaying function

$$
\chi_{\mathrm{fit}}(t)=\chi_{0}+\sum_{i=1}^{3} \chi_{i} e^{-\left(t-t_{0}\right) / t_{i}} .
$$

The three characteristic times involved here are related to each part of the purity, namely, the initial falloff $\left(t_{1}\right)$, the intermediate turn $t_{2}$ and slowly decaying tail $t_{3}$. Furthermore, all exponentials include a reference onset time $t_{0}$, the same for all, and the expression also considers a baseline $\chi_{0}$, which gives the asymptotic value $\chi_{\infty}$. The trend exhibited by the three times in the case of one single signal is displayed in Fig. 10(b) in terms of $x_{0}$. As it can be noticed, while $t_{1}$ and $t_{2}$ are nearly constant in the whole interval, $t_{3}$ undergoes a remarkable increase as we move towards $x_{0} \sim 8-9$ and then it decreases again. This is because of the larger number of modes involved in the superposition, with nearly similar weights, which are thus able to support the coherence for longer times. In the case of two input signals, shown in Fig. 10(c), the trend is less clear, although times are much shorter. Yet it can be noticed that except for a particular value of $x_{0}$ around 8 , where we observe an important decrease in the three times, although especially in $t_{3}$, in all other cases the trend is relatively smooth. If in the single signal case the maximum for $t_{3}$ was associated with a rather complex contribution of modes, the dip here can be justified because the addition of a mirror-symmetric signal removes many of those contributions, thus generating a rather simple superposition, much simpler than for other neighboring values of $x_{0}$.

In order to find an explanation for the different timescales ruling each decay range of the purity, let us consider the energy correlation matrix for several single and double input signals (i.e., in terms of $x_{0}$ ), which corresponds to the density matrix at $t=0$, with elements $\rho_{\alpha \alpha^{\prime}}(0)=c_{\alpha} c_{\alpha^{\prime}}^{*}$. These correlations matrices are displayed in Fig. 11 for single input signals [Fig. 11(a)-Fig. 11(d)] and double input signals [Fig. 11(e)-Fig. 11(h)] for different values of $x_{0}$. The populations $\left|c_{\alpha}\right|^{2}$ of each contributing mode are also shown in the inset of each panel. Consider first a single input signal depending on the spatial symmetry displayed 

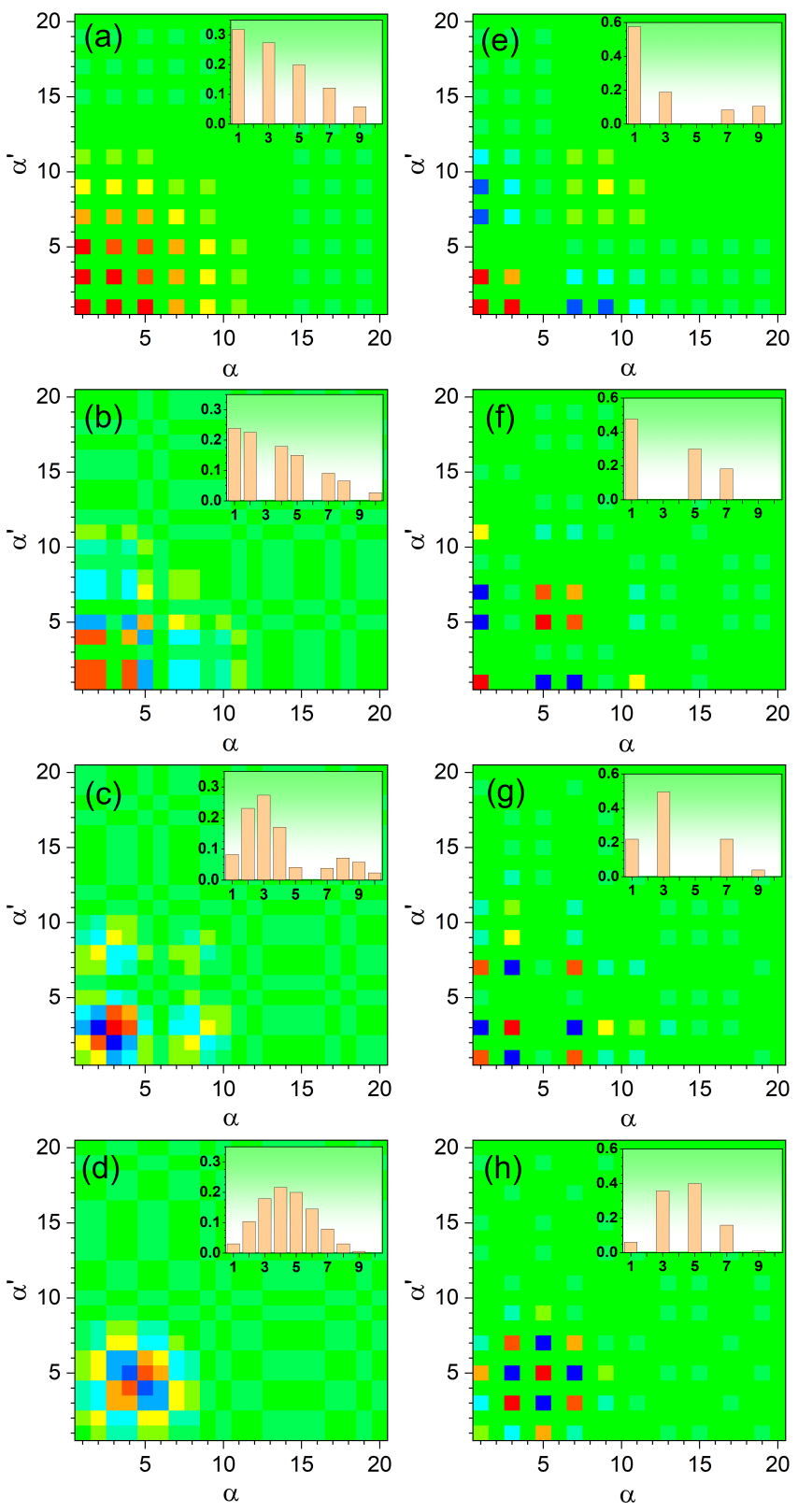

FIG. 11. Energy correlation matrix for single (left column) and double (right column) input signals. For a single input signal (a) $x_{0}=0$, (b) $x_{0}=8.3$, (c) $x_{0}=16.5$, and (d) $x_{0}=20$. For a double input signal (e) $x_{0}=5$, (f) $x_{0}=8.3$, (g) $x_{0}=15$, and $(\mathrm{h}) x_{0}=20$. The color scale ranges from red denoting higher values (greater than or equal to 0.3) to blue for lower ones (less than or equal to-0.3). The panel of each inset indicates the population associated with each particular input state. In all cases, $m=1, \hbar=1, L=50, w=10$ and $\gamma=2 / 5 \pi$. All quantities are given in arbitrary units.

by the single input signal as $x_{0}$ increases, although in a particular manner. Up to nearly $x_{0}=8$, the pattern is analogous to the one shown in the inset of Fig. 11(a), with a prominent presence of odd modes and residual in the case of the even ones (or even zero for $x_{0}=0$ ), but increasing with $x_{0}$. Accordingly, the correlations between neighboring modes increases with $x_{0}$, which also leads to an increase of the long-term time $t_{3}$, as seen in Fig. 10(a). Then, between $x_{0}=8$ and 9 , approximately, we observe paired modes with a lack or residual presence of correlation between each pair, as can be seen in the inset of Fig. 11(b). Because the members of each pair contribute approximately the same, the correlation between them is going to be rather strong, which leads to a maximum in the long-term time. Finally, as $x_{0}$ increases beyond $x_{0}=9$, more contributions start appearing, increasing the amount of first, second, third, etc., member correlations, which eventually translates into a fall in $t_{3}$. A similar behavior is also observed in the case of two input signals, although this time, because of symmetry considerations, there are two positions of particular interest, one for $x_{0}=L / 6 \approx 8.3$, where there are no contributions for $n=2,3$, and 4 , thus implying a strong decay in the correlation matrix, as seen in Fig. 11(f). The same happens for $x_{0}=15$, although this time it is the contribution from the $n=4,5$ and 6 modes that vanishes, which has an effect on the $t_{1}$ and $t_{2}$ times, as seen in Fig. 10(b).

\section{FINAL REMARKS}

Although decoherence is commonly regarded as the mechanism by means of which quantum systems lose their quantumness, thus giving rise to the emergence of a classical world, the quantum world is never abandoned. Decoherence is possible because quantum systems interact with other quantum systems and, as a consequence of such an interaction, when those other surrounding (quantum) systems, known as the environment, are neglected, the behavior of the system of interest exhibits classicaltype features. This has given rise in the literature to a series of different phenomenological or effective decoherence models that try to reproduce the effects of the environment over the system of interest without explicitly specifying the particular dynamics displayed by each environmental system. This is what we generally call the theory of open quantum systems [1, which is in reality a compendium of effective theories with common grounds that allow us to go from one to another.

Such theories or models essentially arise from the observation of the behavior displayed by a quantum system when it is acted upon by an environment. An immediate effect is the gradual loss of interference features (loss of fringe visibility), which will be faster or slower depending on the strength of the system-environment interaction. In this regard, quantum carpets become ideal systems to probe and to evidence the effects of decoherence [48, 49, since the intricate carpet-type patterns exhibited inside a box (a wave guide, regardless of whether the wave is made of light, electrons, or neutrons) going to be very sensitive to an external action. In this work we have explored these effects by considering a simple but insightful coherence damping model based on how more complicated models act on superpositions of energy states and 
also on continuous-variable states. This model describes the action of entangling the system generating the carpets here with another analogous system, which, when it is traced over, gives rise to the exponential decay here observed.

What is interesting here is the fact that, because dissipation (thermalization) effects are not included, a full loss of coherence does not correspond to spatial localization, as one might expect a priori. Rather, it is seen that the annihilation of the coherence terms in the energy density matrix gives rise to a sort of spreading of energy all along the cavity considered, with some concentration around the center of the input signal and its mirrorsymmetric position with respect to $x=0$, or around the center in the case of two symmetrically distributed input signals (initial coherent superpositions of two localized states). This seemingly unusual long-time limit is actually a product of the bare sum of the densities associated with each contributing eigenmode. From this point of view, the oscillatory pattern-type behavior is only a manifestation of how the coherence terms modulate such a bare backbone. When this effect is visualized in terms of Bohmian trajectories, a transition from a highly oscillatory motion to a rather smooth behavior is observed, with a long-time limit being described by motionless trajectories. This is a nice manifestation of the important connection between Bohmian mechanics and an underlying locally varying phase field: As soon as the phase field does not change any more, all Bohmian motion disappears. This situation resembles the widely known stationarity associated with non-degenerate eigenvalues, which has been debated for a long time in the literature 63. It is worth mentioning that at present these behaviors, fully based on Markovian considerations, are being investigated also in terms of a more robust twoparty entanglement model. It is expected that the model will render in a natural fashion the damping factor in positions, but also a counterpart in the energy representation, thus offering an alternative perspective on the coherence transference between parties and its role in decoherence processes.

Another interesting feature that has been observed is that not only the coherence among different energy eigenstates must disappear in order to faithfully reproduce the effects of decoherence. If instead of the probability density we look at the real part of the density matrix (the imaginary part is valid as well), we notice that important structures out of the main diagonal still persist. The reason is that the removal of these contributions requires an additional element related to the loss of point-to-point space correlations, which cannot be readily seen in more refined models. When we introduce a damping term in this direction, all contributions out of the main diagonal gradually disappear. Note that this is a feature that cannot be observed by simply inspecting the probability density, because although interferential traits are seen to disappear with time, the same is not seen at all in the density matrix in space, since all those correlations disappear when we set $x=x^{\prime}$.

Furthermore, in order to determine in a more quantitative manner the effectiveness of decoherence in terms of the initial input state considered, analysis of the purity and the density matrix in the energy representation has also been carried out. In this regard it is worth mentioning that, when the input states have an even parity, the loss of coherence takes place more rapidly than in the case of asymmetric states due to the many more cavity modes that such states can be projected on. To quantify the effect, we analyzed the time scales involved in each case, finding that there are three decay time-scales ruling the behavior of the purity in the short, medium, and long terms, respectively.

Finally, it is worth emphasizing that the analysis presented here, which includes different supplementary tools (densities in space and momentum, density matrices and Bohmian trajectories), can be equally applied to confinement of matter waves and to light pulses pumped into resonant cavities or multimode interference devices, where the generation of twin pulses is based on the phenomenon of recurrences. In these cases, taking advantage of the isomorphism between the Schrödinger equation and the paraxial Helmholtz equation 64, the full analysis presented here for a matter wave could be profitably switched to the analysis of confined light.

\section{ACKNOWLEDGMENTS}

Financial support from the Agencia Estatal de Investigación (AEI) and the European Regional Development Fund (ERDF) through Grant No. FIS2016-76110-P is acknowledged.
[1] H.-P. Breuer and F. Petruccione, The Theory of Open Quantum Systems (Oxford University Press, Oxford, 2002).

[2] E. Joos and H. D. Zeh, The emergence of classical properties through interaction with the environment, Z. Phys. B 59, 223 (1985).

[3] W. H. Zurek, Decoherence and the transition from quantum to classical, Phys. Today 44(10), 36 (1991).

[4] W. H. Zurek, Decoherence and the transition from quan- tum to classical-Revisited, Los Alamos Science 27, 2 (2002).

[5] P. Blanchard and R. Olkiewicz, Decoherence induced transition from quantum to classical dynamics, Rev. Math. Phys. 15, 217 (2003).

[6] D. Giulini, E. Joos, C. Kiefer, J. Kupsch, I.-O. Stamatescu, and H. D. Zeh, Decoherence and the Appearance of a Classical World in Quantum Theory, 2nd ed. (Springer, 1996, Berlin). 
[7] M. Schlosshauer, Decoherence, the measurement problem, and interpretations of quantum mechanics, Rev. Mod. Phys. 76, 1267 (2004).

[8] M. Schlosshauer, Decoherence and the Quantum-toClassical Transition (Springer, Berlin, 2007).

[9] M. Schlosshauer, Quantum decoherence, Phys. Rep. 831, 1 (2019).

[10] A. V. Khaetskii, D. Loss, and L. Glazman, Electron spin decoherence in quantum dots due to interaction with nuclei, Phys. Rev. Lett. 88, 186802 (2002).

[11] A. Mathew and M. K. Nandy, Decoherence study of electron spin states in quantum dots using a simplistic model, Mod. Phys. Lett. B 27, 1350119 (2013).

[12] M. V. Altaisky, N. N. Zolnikova, N. E. Kaputkina, V. A. Krylov, Y. E. Lozovik, and N. S. Dattani, Decoherence and entanglement simulation in a model of quantum neural wetwork based on quantum dots, EPJ Web of Conferences 108, 02006 (2016).

[13] A. P. Flitney and D. Abbott, Quantum games with decoherence, J. Phys. A: Math. Gen. 38, 449 (2005).

[14] L. K. Chen, H. L. Ang, D. Kiang, L. C. Kwek, and C. F. Lo, Quantum prisoner dilemma under decoherence, Phys. Lett. A 316, 317 (2003).

[15] V. Kendon and B. Tregenna, Decoherence can be useful in quantum walks, Phys. Rev. A 67, 042315 (2003).

[16] Y. Yin, D. E. Katsanos, and S. N. Evangelou, Quantum walks on a random environment, Phys. Rev. A 77, 022302 (2008).

[17] G. J. Milburn, Intrinsic decoherence in quantum mechanics, Phys. Rev. A 44, 5401 (1991).

[18] K. Leman, Decoherence induced by discontinuous and stochastic unitary evolution of qubits in quantum computers, Commun. Theor. Phys. 29, 169 (1998).

[19] K. Kimm and H. H. Kwon, Decoherence of the quantum gate in Milburn's model of decoherence, Phys. Rev. A 65, 022311 (2002).

[20] Y. L. Wu, D. L. Deng, X. P. Li, and S. D. Sarma, Intrinsic decoherence in isolated quantum systems, Phys. Rev. B 95, 014202 (2017).

[21] M. Ziman and V. Bužek, All (qubit) decoherences: Complete characterization and physical implementation, Phys. Rev. A 72, 022110 (2005).

[22] A. Czerwinski, Applications of the stroboscopic tomography to selected 2-level decoherence models, Int. J. Theor. Phys. 55, 658 (2016).

[23] I. Floss, C. Lemell, K. Yabana, and J. Burgdörfer, Incorporating decoherence into solid-state time-dependent density functional theory, Phys. Rev. B 99, 224301 (2019).

[24] S. Longhi, Quantum simulation of decoherence in optical waveguide lattices, Opt. Lett. 38, 4884 (2013).

[25] C. Chen and Y. B. Gao, Quantum decoherence of charge qubit coupled to nonlinear nanomechanical resonator, Commun. Theor. Phys. 60, 531 (2013).

[26] S. G. Dlamini, J. T. Francis, X. Zhang, Ş. K. Özdemir, S. N. Chormaic, F. Petruccione, and M. S. Tame, Probing decoherence in plasmonic waveguides in the quantum regime, Phys. Rev. Appl. 9, 024003 (2018).

[27] C. Schroll, W. Belzig, and C. Bruder, Decoherence of cold atomic gases in magnetic microtraps, Phys. Rev. A 68, 043618 (2003).

[28] S. Dehdashti, A. Mahdifar, M. B. Harouni, and R. Roknizadeh, Decoherence of spin-deformed bosonic model, Ann. Phys. (N.Y.) 334, 321 (2013).

[29] L.-M. Duan and G.-C. Guo, Scheme for reducing decoherence in quantum computer memory by transformation to the coherence-preserving states, Chinese Phys. Lett. 14, 488 (1997).

[30] L. M. Duan and G. C. Guo, Reducing decoherence in quantum-computer memory with all quantum bits coupling to the same environment, Phys. Rev. A 57, 737 (1998).

[31] L. M. Duan and G. C. Guo, Quantum error correction with spatially correlated decoherence, Phys. Rev. A 59, 4058 (1999).

[32] P. W. Shor, Scheme for reducing decoherence in quantum computer memory, Phys. Rev. A 52, R2493 (1995).

[33] L. Viola and S. Lloyd, Dynamical suppression of decoherence in two-state quantum systems, Phys. Rev. A 58, 2733 (1998).

[34] E. Novais and H. U. Baranger, Decoherence by correlated noise and quantum error correction, Phys. Rev. Lett. 97, 040501 (2006).

[35] R. Alicki, A unified picture of decoherence control, Chem. Phys. 322, 75 (2006).

[36] C. U. Lei and W.-M. Zhang, Decoherence suppression of open quantum systems through a strong coupling to nonMarkovian reservoirs, Phys. Rev. A 84, 052116 (2011).

[37] Y. S. Kim, J. C. Lee, O. Kwon, and Y. H. Kim, Protecting entanglement from decoherence using weak measurement and quantum measurement reversal, Nature Phys. 8, 117 (2012).

[38] Q. Bi, Quantum computation in triangular decoherencefree subdynamic space, Front. Phys. 10, 198 (2015).

[39] M. Ahsan and S. A. Z. Naqvi, Performance of topological quantum error correction in the presence of correlated noise, Quantum Inf. Comput. 18, 743 (2018).

[40] T. C. Ralph and J. Pienaar, Entanglement decoherence in a gravitational well according to the event formalism, New J. Phys. 16, 085008 (2014).

[41] P. J. Beierle, L. Zhang, and H. Batelaan, Experimental test of decoherence theory using electron matter waves, New J. Phys. 20, 113030 (2018).

[42] S. K. Joshi, J. Pienaar, T. C. Ralph, L. Cacciapuoti, W. McCutcheon, J. Rarity, D. Giggenbach, J. G. Lim, V. Makarov, I. Fuentes, T. Scheidl, E. Beckert, M. Bourennane, D. E. Bruschi, A. Cabello, J. Capmany, A. Carrasco-Casado, E. Diamanti, M. Dušek, D. Elser, A. Gulinatti, R. H. Hadfield, T. Jennewein, R. Kaltenbaek, M. A. Krainak, H. K. Lo, C. Marquardt, G. Milburn, M. Peev, A. Poppe, V. Pruneri, R. Renner, C. Salomon, J. Skaar, N. Solomos, M. Stipčevic, J. P. Torres, M. Toyoshima, P. Villoresi, I. Walrnsley, G. Weihs, H. Weinfurter, A. Zeilinger, M. Zukowski, and R. Ursin, Space QUEST mission proposal: Experimentally testing decoherence due to gravity, New J. Phys. 20, 063016 (2018).

[43] P. Xu, Y. Q. Ma, J. G. Ren, H. L. Yong, T. C. Ralph, S. K. Liao, J. Yin, W. Y. Liu, W. Q. Cai, X. Han, H. N. Wu, W. Y. Wang, F. Z. Li, M. Yang, F. L. Lin, L. Li, N. L. Liu, Y. A. Chen, C. Y. Lu, Y. B. Chen, J. Y. Fan, C. Z. Peng, and J. W. Pan, Satellite testing of a gravitationally induced quantum decoherence model, Science 366, 132 (2019).

[44] A. E. Kaplan, P. Stifter, K. A. H. van Leeuwen, W. E. Lamb, Jr., and W. P. Schleich, Intermode traces Fundamental interference phenomenon in quantum and 
wave physics, Phys. Scr. T76, 93 (1998)

[45] I. Marzoli, F. Saif, I. Bialynicki-Birula, O. M. Friesch, A. E. Kaplan, and W. P. Schleich, Quantum carpets made simple, Acta Phys. Slovaca 48, 323 (1998).

[46] A. E. Kaplan, I. Marzoli, W. E. Lamb, Jr., and W. P. Schleich, Multimode interference: Highly regular pattern formation in quantum wave-packet evolution, Phys. Rev. A 61, 032101 (2000).

[47] M. Berry, I. Marzoli, and W. Schleich, Quantum carpets, carpets of light, Phys. World 14(6), 39 (2001).

[48] R. Bonifacio, I. Marzoli, and W. P. Schleich, Nondissipative decoherence for quantum carpets, J. Mod. Optic. 47, 2891 (2000).

[49] P. Kazemi, S. Chaturvedi, I. Marzoli, R. F. O'Conell, and W. P. Schleich, Quantum carpets: A tool to observe decoherence, New J. Phys. 15, 013052 (2013).

[50] A. S. Sanz, Effective Markovian description of decoherence in bound systems, Can. J. Chem. 92, 168 (2014).

[51] A. S. Sanz and F. Borondo, A quantum trajectory description of decoherence, Eur. Phys. J. D 44, 319 (2007).

[52] A. S. Sanz and F. Borondo, Contextuality, decoherence and quantum trajectories, Chem. Phys. Lett. 478, 301 (2009).

[53] A. Luis and A. S. Sanz, What dynamics can be expected for mixed states in two-slit experiments?, Ann. Phys. (N.Y.) 357, 95 (2015).

[54] A. S. Sanz and S. Miret-Artés, A trajectory-based understanding of quantum interference, J. Phys. A: Math. Theor. 41, 435303 (2008).

[55] A. S. Sanz, Bohm's approach to quantum mechanics: Alternative theory or practical picture?, Front. Phys. 14, 11301 (2019).

[56] W. K. Burns and A. F. Milton, An analytic solution for mode coupling in optical waveguide branches, IEEE J. Quantum Electron. QE-16, 446 (1980).

[57] L. B. Soldano and E. C. M. Pennings, Optical multimodel interference devices based on self-imaging: Principles and applications, J. Light. Technol. 13, 615 (1995).

[58] L. A. Coldren, S. W. Corzine, and M. L. Mašanović, Diode Lasers and Photonic Integrated Circuits, 2nd Ed. (Wiley, Hoboken, NJ, 2012).

[59] E. Andersson, T. Calarco, R. Folman, M. Andersson, B. Hessmo, and J. Schmiedmayer, Multimode interferometer for guided matter waves, Phys. Rev. Lett. 88, 100401 (2002).
[60] J. Tounli, A. Alvarado, and A. S. Sanz, Boundary bound diffraction: A combined spectral and bohmian mechanics, Phys. Scr. 94, 035202 (2019).

[61] By referring to the localized state entering the cavity as the input signal, the connection to the language employed in fiber optic communications becomes closer, particularly in the realm of quantum optics with single-photon transmission, where this type of scenario could be experimentally tested.

[62] L. I. Schiff, Quantum Mechanics, 3rd Ed. (McGraw-Hill, Singapore, 1968).

[63] P. R. Holland, The Quantum Theory of Motion (Cambridge University Press, Cambridge, 1993).

[64] A. S. Sanz, J. Campos-Martínez, and S. Miret-Artés, Transmission properties in waveguides: An optical streamline analysis, J. Opt. Am. Soc. A 29, 695 (2012).

[65] A. S. Sanz and S. Miret-Artés, A Trajectory Description of Quantum Processes. I. Fundamentals, Lecture Notes in Physics, Vol. 850 (Springer, Berlin, 2012).

[66] J. Tounli and A. S. Sanz, (unpublished).

[67] A. S. Sanz, F. Borondo, and S. Miret-Artés, Particle diffraction studied using quantum trajectories, J. Phys.: Condens. Matter 14, 6109 (2002).

[68] R. Omnès, Consistent interpretations of quantum mechanics, Rev. Mod. Phys. 64, 339 (1992).

[69] E. Joos, Decoherence and the Appearance of a Classical World in Quantum Theory (Springer, Berlin, 1996), Chap. 3, pp. 41-180, 2nd Ed.

[70] Y. Elran and P. Brumer, Decoherence in an anharmonic oscillator coupled to a thermal environment: A semiclassical forward-backward approach, J. Chem. Phys. 121, 2673 (2004).

[71] Y. Elran and P. Brumer, Quantum decoherence of $\mathrm{I}_{2}$ in liquid xenon: A classical Wigner approach, J. Chem. Phys. 138, 234308 (2013).

[72] A. S. Sanz and S. Miret-Artés, A causal look into the quantum Talbot effect, J. Chem. Phys. 126, 234106 (2007).

[73] H. Wang, M. Thoss, K. L. Sorge, R. Gelabert, X. Giménez, and W. H. Miller, Semiclassical description of quantum coherence effects and their quenching: A forward-backward initial value representation study, J. Chem. Phys. 114, 2562 (2001). 